\title{
Quasilinear vector differential equations with maximal monotone terms and nonlinear boundary conditions
}

\author{
by Ralf Bader (Münich) and Nikolaos S. Papageorgiou (Athens)
}

\begin{abstract}
We consider a quasilinear vector differential equation which involves the $p$-Laplacian and a maximal monotone map. The boundary conditions are nonlinear and are determined by a generally multivalued, maximal monotone map. We prove two existence theorems. The first assumes that the maximal monotone map involved is everywhere defined and in the second we drop this requirement at the expense of strengthening the growth hypothesis on the vector field. The proofs are based on the theory of operators of monotone type and on the Leray-Schauder fixed point theorem. At the end we present some special cases (including the classical Dirichlet, Neumann and periodic problems), which illustrate the general and unifying features of our work.
\end{abstract}

1. Introduction. In this paper we study the following nonlinear boundary value problem in $\mathbb{R}^{N}$ :

$$
\left\{\begin{array}{l}
\left(\left\|x^{\prime}(t)\right\|^{p-2} x^{\prime}(t)\right)^{\prime} \in A(x(t))+f\left(t, x(t), x^{\prime}(t)\right) \quad \text { a.e. on } T=[0, b] \\
\left(\varphi\left(x^{\prime}(0)\right),-\varphi\left(x^{\prime}(b)\right)\right) \in \xi(x(0), x(b)), \quad 2 \leq p<\infty .
\end{array}\right.
$$

Here $A: \mathbb{R}^{N} \rightarrow 2^{\mathbb{R}^{N}}$ is a maximal monotone operator and $\varphi: \mathbb{R}^{N} \rightarrow \mathbb{R}^{N}$ is defined by $\varphi(r)=\|r\|^{p-2} r$.

Recently nonlinear boundary value problems involving the $p$-Laplacian have been studied by several authors. We mention the works of BoccardoDrábek-Giachetti-Kučera [2], Dang-Oppenheimer [3], del Pino-ElguetaManásevich [4], del Pino-Manásevich-Murúa [5], Fabry-Fayyad [6], Guo [7], Kandilakis-Papageorgiou [11] and Manásevich-Mawhin [12]. In all these papers $A \equiv 0$ and with the exception of Manásevich-Mawhin, all the other

2000 Mathematics Subject Classification: Primary 34B15.

Key words and phrases: monotone operator, maximal monotone operator, demicontinuous operator, coercive operator, resolvent operator, Yosida approximation, projection theorem, measurable selection, Dirichlet, Neumann and periodic problems.

This work was done while the first author visited NTUA. Support was provided by a scholarship offered by Deutsche Forschungsgemeinschaft (DFG), Bonn (Germany). 
works deal with the scalar problem (i.e. $N=1$ ). It should be mentioned that Dang-Oppenheimer (scalar problem) and Manásevich-Mawhin (vector problem) work with a more general $p$-Laplacian-like differential operator. Boccardo-Drábek-Giachetti-Kučera and del Pino-Elgueta-Manásevich deal with the Dirichlet problem, del Pino-Manásevich-Murúa, Fabry-Fayyad and Manásevich-Mawhin examine the periodic problem and the first of these three works proves a multiplicity result, Guo considers both the periodic and the Neumann problem, Kandilakis-Papageorgiou consider the Neumann problem and finally Dang-Oppenheimer investigate all three problems (Dirichlet, Neumann and periodic problem). Also in the works of Boccardo-Drábek-Giachetti-Kučera, del Pino-Elgueta-Manásevich, del Pino-Manásevich-Murúa, Fabry-Fayyad, Guo (the Neumann problem) and Kandilakis-Papageorgiou, the right hand side function $f$ is independent of $x^{\prime}$. Most of these works use degree-theoretic techniques, while Kandilakis-Papageorgiou base their proof on variational arguments (critical point theory for nonsmooth functionals, since they do not assume continuity of $f(t, \cdot))$.

Our formulation here unifies the basic vector boundary value problems (Dirichlet, Neumann and periodic problem) and goes beyond them. Also the presence in (1) of the maximal monotone multivalued operator $A$ incorporates second order systems with convex potential. Such systems were studied by Mawhin-Willem [14] (see Section 1.7). In the book of Mawhin-Willem the potential function is nonautonomous, but smooth. The fact that in our case $A$ can be multivalued allows us to include also problems with nonsmooth (convex) potential. Moreover, in Theorem 6 we do not require that $\operatorname{dom} A=\left\{x \in \mathbb{R}^{N}: A(x) \neq \emptyset\right\}=\mathbb{R}^{N}$ and so our formulation incorporates second order variational inequalities (see Example (a), Section 4).

Our method of proof is based on the theory of operators of monotone type, which leads to an eventual application of the Leray-Schauder fixed point theorem.

2. Preliminaries. Since our approach uses the theory of nonlinear operators of monotone type, in this section we recall some basic definitions and facts from this theory that we will need. The basic references are the books of $\mathrm{Hu}-$ Papageorgiou [10] and Zeidler [16].

Let $X$ be a reflexive Banach space and $X^{*}$ its topological dual. A possibly multivalued map $A: D \subseteq X \rightarrow 2^{X^{*}}$ is said to be monotone if for all $x, y \in D$ and all $x^{*} \in A(x), y^{*} \in A(y)$, we have $\left(x^{*}-y^{*}, x-y\right) \geq 0$, where $(\cdot, \cdot)$ denotes the duality brackets for the pair $\left(X, X^{*}\right)$. If $\left(x^{*}-y^{*}, x-y\right)=0$ implies $x=y$, then we say that $A$ is strictly monotone. A monotone map for which the inequalities $\left(x^{*}-y^{*}, x-y\right) \geq 0$ for all $y \in D$ and all $y^{*} \in A(y)$ imply $x \in D$ and $x^{*} \in A(x)$ is said to be maximal monotone. It is clear 
from this definition that $A$ is maximal monotone if and only if its graph Gr $A=\left\{\left[x, x^{*}\right] \in X \times X^{*}: x^{*} \in A(x)\right\}$ is maximal with respect to inclusion among the graphs of monotone maps. If $A$ is maximal monotone, then for any $x \in D$, the set $A(x)$ is nonempty, closed and convex. Moreover, $\operatorname{Gr} A$ is demiclosed, i.e. if $\left[x_{n}, x_{n}^{*}\right] \in \mathrm{Gr} A, n \geq 1$, and either $x_{n} \rightarrow x$ in $X$ and $x_{n}^{*} \stackrel{w}{\rightarrow} x^{*}$ in $X^{*}$, or $x_{n} \stackrel{w}{\rightarrow} x$ in $X$ and $x_{n}^{*} \rightarrow x^{*}$ in $X^{*}$, then $\left[x, x^{*}\right] \in \mathrm{Gr} A$.

Let $D=X$ and assume that $A: X \rightarrow X^{*}$ is single-valued. We say that $A$ is demicontinuous if $x_{n} \rightarrow x$ in $X$ implies $A\left(x_{n}\right) \stackrel{w}{\rightarrow} A(x)$, i.e. $A$ is sequentially continuous from $X$ into $X_{w}^{*}$ (here $X_{w}^{*}$ denotes the Banach space $X^{*}$ equipped with the weak topology). A monotone, demicontinuous map $A$ : $X \rightarrow X^{*}$ is maximal monotone. A map $A: D \subseteq X \rightarrow 2^{X^{*}}$ is said to be coercive if $D$ is bounded or $D$ is unbounded and $\inf \left\{\left\|x^{*}\right\|_{*}: x^{*} \in A(x)\right\} \rightarrow \infty$ as $\|x\| \rightarrow \infty, x \in D$ (here $\|\cdot\|$ and $\|\cdot\|_{*}$ are the norms of $X$ and $X^{*}$ respectively). A basic theorem says that a maximal monotone, coercive operator is surjective. In particular, a monotone, demicontinuous coercive map $A: X \rightarrow X^{*}$ is surjective.

Suppose $X^{*}$ is strictly convex and $A: D \subseteq X \rightarrow 2^{X^{*}}$ is maximal monotone. We know that for every $x \in D, A(x)$ is nonempty, closed and convex. Thus we can define the single-valued map $A^{0}: D \subseteq X \rightarrow X^{*}$ by $A^{0}(x)=\operatorname{proj}(0 ; A(x))$, i.e. $A^{0}(x)$ is the unique element of $A(x)$ with minimal norm. We call $A^{0}$ the minimal section; it is important in the theory of maximal monotone operators. Recall that a reflexive Banach space can be equivalently renormed so that both $X$ and $X^{*}$ are strictly convex (Asplund's theorem, see Hu-Papageorgiou [10]). If $X=H=$ Hilbert space, $A: D \subseteq H \rightarrow 2^{H}$ is maximal monotone and $\lambda>0$, we introduce $J_{\lambda}=(I+\lambda A)^{-1}$ (the resolvent of $A$ ) and $A_{\lambda}=\lambda^{-1}\left(I-J_{\lambda}\right)$ (the Yosida approximation of $A$ ). We have $\operatorname{dom} J_{\lambda}=\operatorname{dom} A_{\lambda}=H$ for all $\lambda>0$. Moreover, for every $\lambda>0, J_{\lambda}$ is nonexpansive (i.e. Lipschitz with constant 1$), A_{\lambda}(x) \in A\left(J_{\lambda}(x)\right)$ for all $x \in H, A_{\lambda}(\cdot)$ is monotone and Lipschitz with constant $\lambda^{-1}$ (hence maximal monotone), and $\left\|A_{\lambda}(x)\right\| \leq\left\|A^{0}(x)\right\|$ for all $x \in D$. In addition, $\lim _{\lambda \rightarrow 0+} A_{\lambda}(x)=A^{0}(x)$ for all $x \in D$ and $\lim _{\lambda \rightarrow 0+} J_{\lambda}(x)=\operatorname{proj}(x ; \bar{D})$ for all $x \in H$. A well known maximal monotone operator is the subdifferential $\partial \varphi$ of a convex, lower semicontinuous, proper (i.e. not identically $+\infty$ ) function $\varphi$. Recall that $\partial \varphi: X \rightarrow 2^{X^{*}}$ is defined by $\partial \varphi(x)=\left\{x^{*} \in X^{*}:\left(x^{*}, y-x\right) \leq \varphi(y)-\varphi(x)\right.$ for all $\left.y \in Y\right\}$. Finally if $C \subseteq X$ is nonempty, then we denote by $\sigma(\cdot, C): X^{*} \rightarrow \overline{\mathbb{R}}=\mathbb{R} \cup\{+\infty\}$ the support function of $C$, i.e. $\sigma\left(x^{*}, C\right)=\sup \left\{\left(x^{*}, c\right): c \in C\right\}$, which is sublinear and weakly lower semicontinuous. So if $C$ is closed and convex, then $C=\left\{x \in X:\left(x^{*}, x\right) \leq \sigma\left(x^{*}, C\right)\right.$ for all $\left.x^{*} \in X^{*}\right\}$.

Let $Y$ and $Z$ be Banach spaces. An operator $K: Y \rightarrow Z$ (not necessarily linear) is said to be: 
(a) completely continuous if $y_{n} \stackrel{w}{\rightarrow} y$ in $Y$ implies $K\left(y_{n}\right) \rightarrow K(y)$ in $Z$;

(b) compact if $K$ is continuous and maps bounded sets in $Y$ into relatively compact sets in $Z$.

In general these two notions are not comparable. However, if $Y$ is reflexive, then complete continuity of $K$ implies compactness. Moreover, if $K$ is linear and $Y$ is reflexive, then the two notions are equivalent.

As already mentioned, our proof ultimately relies on a fixed point argument which makes use of the "Leray-Schauder fixed point theorem". For the convenience of the reader we recall this result here (see Zeidler [15], Theorem 6A, p. 245).

Theorem 1. If $Y$ is a Banach space, $K: Y \rightarrow Y$ is compact and there exists $r>0$ such that $y=\lambda K(y)$ with $0<\lambda<1$ implies $\|y\| \leq r(a$ priori bound), then $K$ has a fixed point (i.e. there exists $y \in Y$ such that $y=K(y))$.

Finally recall that if $2 \leq p<\infty$ and $a, c \in \mathbb{R}$, then $2^{2-p}|a-c|^{p} \leq$ $\left(|a|^{p-2} a-|c|^{p-2} c\right)(a-c)$.

Our hypotheses on the data of (1) are the following:

$\mathrm{H}(A)_{1} . \quad A: \mathbb{R}^{N} \rightarrow 2^{\mathbb{R}^{N}}$ is a maximal monotone map such that $\operatorname{dom} A=$ $\left\{x \in \mathbb{R}^{N}: A(x) \neq \emptyset\right\}=\mathbb{R}^{N}$ and $0 \in A(0)$.

Remark. The hypothesis $0 \in A(0)$ is not an essential restriction, since we can always have it by translating things if necessary. Also since $\operatorname{dom} A=$ $\mathbb{R}^{N}, A^{0}(\cdot)$ is bounded on compact subsets of $\mathbb{R}^{N}$.

We can weaken the conditions on $A$ (at the expense of strengthening the growth hypothesis on $f(t, x, \cdot))$ as follows:

$\mathrm{H}(A)_{2} . \quad A: \mathbb{R}^{N} \rightarrow 2^{\mathbb{R}^{N}}$ is a maximal monotone map such that $0 \in A(0)$.

Remark. Again it is enough to assume that $0 \in \operatorname{dom} A$ and by translation we will have $0 \in A(0)$.

$\mathrm{H}(f)_{1} . \quad f: T \times \mathbb{R}^{N} \times \mathbb{R}^{N} \rightarrow \mathbb{R}^{N}$ is a function such that

(i) for all $(x, y) \in \mathbb{R}^{N} \times \mathbb{R}^{N}, t \mapsto f(t, x, y)$ is measurable;

(ii) for almost all $t \in T,(x, y) \mapsto f(t, x, y)$ is continuous;

(iii) for almost all $t \in T$ and all $x, y \in \mathbb{R}^{N}$,

$$
(f(t, x, y), x)_{\mathbb{R}^{N}} \geq-a\|x\|^{p}-\gamma\|x\|^{r}\|y\|^{p-r}-c(t)\|x\|^{s}
$$

with $a, \gamma \geq 0,1 \leq r, s<p$ and $c \in L^{1}(T)$;

(iv) there exists $M>0$ such that if $\left\|x_{0}\right\|>M$ and $\left(x_{0}, y_{0}\right)_{\mathbb{R}^{N}}=0$, then we can find $\delta>0$ and $\xi>0$ such that for almost all $t \in T$,

$$
\inf \left[(f(t, x, y), x)_{\mathbb{R}^{N}}+\|y\|^{p}:\left\|x-x_{0}\right\|+\left\|y-y_{0}\right\|<\delta\right] \geq \xi>0 ;
$$


(v) for almost all $t \in T$ and all $x, y \in \mathbb{R}^{N}$,

$$
\|f(t, x, y)\| \leq \gamma_{1}(t,\|x\|)+\gamma_{2}(t,\|x\|)\|y\|^{p-1}
$$

with $\sup _{0 \leq r \leq k} \gamma_{1}(t, r) \leq \eta_{1, k}(t)$ a.e. on $T, \eta_{1, k} \in L^{q}(T)(1 / p+1 / q=1)$ and $\sup _{0 \leq r \leq k} \gamma_{2}(t, r) \leq \eta_{2, k}(t)$ a.e. on $T, \eta_{2, k} \in L^{\infty}(T)$.

Remark. Hypothesis $\mathrm{H}(f)$ (iv) is a slight extension of the classical Nagumo-Hartman condition for continuous vector fields (see Hartman [8], p. 433).

When $\operatorname{dom} A \neq \mathbb{R}^{N}$, we have to strengthen the growth condition on $f(t, x, \cdot)$. More precisely, we will need the following hypothesis:

$\mathrm{H}(f)_{2} . \quad f: T \times \mathbb{R}^{N} \times \mathbb{R}^{N} \rightarrow \mathbb{R}^{N}$ is a function that satisfies (i)-(iv) of $\mathrm{H}(f)_{1}$ and

(v) for almost all $t \in T$ and all $x, y \in \mathbb{R}^{N}$, we have

$$
\|f(t, x, y)\| \leq \gamma_{1}(t,\|x\|)+\gamma_{2}(t,\|x\|)\|y\|
$$

with $\sup _{0 \leq r \leq k} \gamma_{1}(t, r) \leq \eta_{1, k}(t)$ a.e. on $T, \eta_{1, k} \in L^{2}(T)$ and $\sup _{0 \leq r \leq k} \gamma_{2}(t, r)$ $\leq \eta_{2, k}(t)$ a.e. on $T, \eta_{2, k} \in L^{2 p /(p-2)}(T)$ (as usual let $r / 0=\infty$ for $r>0$ ).

$\mathrm{H}(\xi) . \quad \xi: \mathbb{R}^{N} \times \mathbb{R}^{N} \rightarrow 2^{\mathbb{R}^{N} \times \mathbb{R}^{N}}$ is a maximal monotone map such that $(0,0) \in \xi(0,0)$ and one of the following holds: or

(i) for every $\left(a^{\prime}, d^{\prime}\right) \in \xi(a, d)$, we have $\left(a^{\prime}, a\right)_{\mathbb{R}^{N}} \geq 0$ and $\left(d^{\prime}, d\right)_{\mathbb{R}^{N}} \geq 0$;

(ii) $\operatorname{dom} \xi=\left\{(a, d) \in \mathbb{R}^{N} \times \mathbb{R}^{N}: a=d\right\}$.

$\mathrm{H}_{0}$. For all $(a, d) \in \operatorname{dom} \xi$ and all $\left(a^{\prime}, d^{\prime}\right) \in \xi(a, d)$,

$$
\left(A_{\lambda}(a), a^{\prime}\right)_{\mathbb{R}^{N}}+\left(A_{\lambda}(d), d^{\prime}\right)_{\mathbb{R}^{N}} \geq 0 \quad \text { for all } \lambda>0 .
$$

REMARK. If $\xi=\partial \psi$ with $\psi: \mathbb{R}^{N} \times \mathbb{R}^{N} \rightarrow \mathbb{R}$ convex (hence locally Lipschitz), then if we denote by $\partial_{i} \psi, i=1,2$, the partial subdifferential of $\psi(a, d)$ with respect to $a$ (resp. $d)$, then $\partial \psi(a, d) \subseteq \partial_{1} \psi(a, d) \times \partial_{2} \psi(a, d)$. In this setting the condition that $\left(A_{\lambda}(a), a^{\prime}\right)_{\mathbb{R}^{N}} \geq 0$ and $\left(A_{\lambda}(d), d^{\prime}\right)_{\mathbb{R}^{N}} \geq$ 0 for all $\left(a^{\prime}, d^{\prime}\right) \in \xi(a, d),(a, d) \in \operatorname{dom} \xi$, is equivalent to saying that $\psi\left(J_{\lambda}(a), d\right) \leq \psi(a, d)$ and $\psi\left(a, J_{\lambda}(d)\right) \leq \psi(a, d)$ respectively (see Hu-Papageorgiou [10]).

3. Auxiliary results. We start by solving the following auxiliary problem:

$$
\left\{\begin{array}{l}
-\left(\left\|x^{\prime}(t)\right\|^{p-2} x^{\prime}(t)\right)^{\prime}+\|x(t)\|^{p-2} x(t)=h(t) \quad \text { a.e. on } T \\
\left(\varphi\left(x^{\prime}(0)\right),-\varphi\left(x^{\prime}(b)\right)\right) \in \xi(x(0), x(b)), \quad 2 \leq p<\infty
\end{array}\right.
$$

Here $h \in L^{q}\left(T, \mathbb{R}^{N}\right)$ and $1 / p+1 / q=1$. By a solution of problem (2) we mean a function $x \in C^{1}\left(T, \mathbb{R}^{N}\right)$ such that $\left\|x^{\prime}(\cdot)\right\|^{p-2} x^{\prime}(\cdot) \in W^{1, q}\left(T, \mathbb{R}^{N}\right)$ and $x$ satisfies (2). 
Proposition 2. If $\xi: \mathbb{R}^{N} \times \mathbb{R}^{N} \rightarrow 2^{\mathbb{R}^{N}} \times \mathbb{R}^{N}$ is a maximal monotone map with $(0,0) \in \xi(0,0)$, then problem (2) has a unique solution $x \in C^{1}\left(T, \mathbb{R}^{N}\right)$.

Pr o of. Given $v, w \in \mathbb{R}^{N}$, we consider the following two-point boundary value problem:

$$
\left\{\begin{array}{l}
-\left(\left\|x^{\prime}(t)\right\|^{p-2} x^{\prime}(t)\right)^{\prime}+\|x(t)\|^{p-2} x(t)=h(t) \quad \text { a.e. on } T \\
x(0)=v, \quad x(b)=w .
\end{array}\right.
$$

Let $\eta(t)=(1-t / b) v+(t / b) w$, so that $\eta(0)=v, \eta(b)=w$. We introduce the function $y(t)=x(t)-\eta(t)$ and rewrite problem (3) as a homogeneous Dirichlet problem for $y$ :

$$
\left\{\begin{array}{l}
-\left(\left\|(y+\eta)^{\prime}(t)\right\|^{p-2}(y+\eta)^{\prime}(t)\right)^{\prime}+\|(y+\eta)(t)\|^{p-2}(y+\eta)(t) \\
y(0)=y(b)=0 .
\end{array}\right.
$$

We solve (4) for $y$ and then $x=y+\eta$ will be the solution of (3). To solve (4), let $V_{1}: W_{0}^{1, p}\left(T, \mathbb{R}^{N}\right) \rightarrow W^{-1, q}\left(T, \mathbb{R}^{N}\right)$ be defined by

$$
\left\langle V_{1}(u), z\right\rangle=\int_{0}^{b}\left\|u^{\prime}+\eta^{\prime}\right\|^{p-2}\left(u^{\prime}+\eta^{\prime}, z^{\prime}\right)_{\mathbb{R}^{N}} d t+\int_{0}^{b}\|u+\eta\|^{p-2}(u+\eta, z)_{\mathbb{R}^{N}} d t
$$

for all $u, z \in W_{0}^{1, p}\left(T, \mathbb{R}^{N}\right)$. Here $\langle\cdot, \cdot\rangle$ are the duality brackets for the pair $\left(W_{0}^{1, p}\left(T, \mathbb{R}^{N}\right), W^{-1, q}\left(T, \mathbb{R}^{N}\right)\right)$. Then for $u, z \in W_{0}^{1, p}\left(T, \mathbb{R}^{N}\right)$, we have

$$
\begin{aligned}
& \left\langle V_{1}(u)-V_{1}(z), u-z\right\rangle \\
& =\int_{0}^{b}\left\|u^{\prime}+\eta^{\prime}\right\|^{p-2}\left(u^{\prime}+\eta^{\prime}, u^{\prime}-z^{\prime}\right)_{\mathbb{R}^{N}} d t+\int_{0}^{b}\|u+\eta\|^{p-2}(u+\eta, u-z)_{\mathbb{R}^{N}} d t \\
& \quad-\int_{0}^{b}\left\|z^{\prime}+\eta^{\prime}\right\|^{p-2}\left(z^{\prime}+\eta^{\prime}, u^{\prime}-z^{\prime}\right)_{\mathbb{R}^{N}} d t-\int_{0}^{b}\|z+\eta\|^{p-2}(z+\eta, u-z)_{\mathbb{R}^{N}} d t .
\end{aligned}
$$

Note that

$$
\begin{aligned}
\int_{0}^{b}\left[\left\|u^{\prime}+\eta^{\prime}\right\|^{p-2}\left(u^{\prime}+\eta^{\prime}, u^{\prime}-z^{\prime}\right)_{\mathbb{R}^{N}}-\left\|z^{\prime}+\eta^{\prime}\right\|^{p-2}\left(z^{\prime}+\eta^{\prime}, u^{\prime}-z^{\prime}\right)_{\mathbb{R}^{N}}\right] d t \\
\quad \geq \int_{0}^{b}\left(\left\|u^{\prime}+\eta^{\prime}\right\|-\left\|z^{\prime}+\eta^{\prime}\right\|\right)\left(\left\|u^{\prime}+\eta^{\prime}\right\|^{p-1}-\left\|z^{\prime}+\eta^{\prime}\right\|^{p-1}\right) d t \\
\geq 2^{2-p} \int_{0}^{b}\left|\left\|u^{\prime}+\eta^{\prime}\right\|-\left\|z^{\prime}+\eta^{\prime}\right\|\right|^{p} d t .
\end{aligned}
$$


Similarly we obtain

$$
\begin{aligned}
\int_{0}^{b}\left[\|u+\eta\|^{p-2}(u+\eta, u-z)_{\mathbb{R}^{N}}-\right. & \left.\|z+\eta\|^{p-2}(z+\eta, u-z)_{\mathbb{R}^{N}}\right] d t \\
& \geq 2^{2-p} \int_{0}^{b}|\|u+\eta\|-\|z+\eta\||^{p} d t .
\end{aligned}
$$

From (5) and (6), we infer that $\left\langle V_{1}(u)-V_{1}(z), u-z\right\rangle \geq 0$, i.e. $V_{1}(\cdot)$ is monotone. In fact, $V_{1}$ is strictly monotone. Indeed, if $\left\langle V_{1}(u)-V_{1}(z), u-z\right\rangle$ $=0$, then

$$
\int_{0}^{b}\left|\left\|u^{\prime}+\eta^{\prime}\right\|-\left\|z^{\prime}+\eta^{\prime}\right\|\right|^{p} d t+\int_{0}^{b}|\|u+\eta\|-\|z+\eta\||^{p} d t=0
$$

hence

$$
\begin{aligned}
\left\|\left(u^{\prime}+\eta^{\prime}\right)(t)\right\| & =\left\|\left(z^{\prime}+\eta^{\prime}\right)(t)\right\|=k_{1}(t), \\
\|(u+\eta)(t)\| & =\|(z+\eta)(t)\|=k_{2}(t)
\end{aligned}
$$

for almost all $t \in T$. So we have

$$
0=\int_{0}^{b} k_{1}(t)^{p-2}\left\|u^{\prime}(t)-z^{\prime}(t)\right\|^{2} d t+\int_{0}^{b} k_{2}(t)^{p-2}\|u(t)-z(t)\|^{2} d t,
$$

thus $u^{\prime}=z^{\prime}$ and $u=z$, and so $V_{1}$ is strictly monotone.

Also using the extended dominated convergence theorem (see for example $\mathrm{Hu}$-Papageorgiou [10], Theorem A.2.54, p. 907), we can easily check that $V_{1}$ is demicontinuous. Moreover,

$$
\begin{aligned}
\left\langle V_{1}(u), u\right\rangle & =\int_{0}^{b}\left\|u^{\prime}+\eta^{\prime}\right\|^{p-2}\left(u^{\prime}+\eta^{\prime}, u^{\prime}\right)_{\mathbb{R}^{N}} d t+\int_{0}^{b}\|u+\eta\|^{p-2}(u+\eta, u)_{\mathbb{R}^{N}} d t \\
& \geq\left\|u^{\prime}+\eta^{\prime}\right\|_{p}^{p}-\left\|u^{\prime}+\eta^{\prime}\right\|_{p}^{p-1}\left\|\eta^{\prime}\right\|_{p}+\|u+\eta\|_{p}^{p}-\|u+\eta\|_{p}^{p-1}\|\eta\|_{p} \\
& \geq\|u+\eta\|_{1, p}^{p}-\mu_{1}\|u+\eta\|_{1, p}^{p-1} \quad \text { for some } \mu_{1}>0 .
\end{aligned}
$$

Here $\|\cdot\|_{1, p}$ denotes the norm of the Sobolev space $W_{0}^{1, p}\left(T, \mathbb{R}^{N}\right)$. So $V_{1}$ is coercive. Therefore $V_{1}$, being monotone, demicontinuous (hence maximal monotone) and coercive, is surjective. So there exists $y \in W_{0}^{1, p}\left(T, \mathbb{R}^{N}\right)$ such that $V_{1}(y)=h$. Evidently by the strict monotonicity of $V_{1}$, this $y$ is unique.

Let $\psi \in C_{0}^{\infty}\left(T, \mathbb{R}^{N}\right)$ and denote by $(\cdot, \cdot)_{p q}$ the duality brackets for the pair $\left(L^{p}\left(T, \mathbb{R}^{N}\right), L^{q}\left(T, \mathbb{R}^{N}\right)\right)$. We have $\left\langle V_{1}(y), \psi\right\rangle=(h, \psi)_{p q}$, hence

$\int_{0}^{b}\left\|y^{\prime}+\eta^{\prime}\right\|^{p-2}\left(y^{\prime}+\eta^{\prime}, \psi^{\prime}\right)_{\mathbb{R}^{N}} d t+\int_{0}^{b}\|y+\eta\|^{p-2}(y+\eta, \psi)_{\mathbb{R}^{N}} d t=\int_{0}^{b}(h, \psi)_{\mathbb{R}^{N}} d t$ and so 


$$
\begin{array}{r}
-\left\langle\left(\left\|y^{\prime}+\eta^{\prime}\right\|^{p-2}\left(y^{\prime}+\eta^{\prime}\right)\right)^{\prime}, \psi\right\rangle+\left\langle\|y+\eta\|^{p-2}(y+\eta), \psi\right\rangle \\
=\langle h, \psi\rangle \quad \text { (by Green's identity). }
\end{array}
$$

Since $\left(\left\|y^{\prime}+\eta^{\prime}\right\|^{p-2}\left(y^{\prime}+\eta^{\prime}\right)\right)^{\prime} \in W^{-1, q}\left(T, \mathbb{R}^{N}\right)$ (see the representation theorem for the space $W^{-1, q}\left(T, \mathbb{R}^{N}\right)$ in Adams [1], Theorem 3.10, p. 50) and since $C_{0}^{\infty}\left(T, \mathbb{R}^{N}\right)$ is dense in the predual space $W_{0}^{1, p}\left(T, \mathbb{R}^{N}\right)$ (recall that $\left.W_{0}^{1, p}\left(T, \mathbb{R}^{N}\right)^{*}=W^{-1, q}\left(T, \mathbb{R}^{N}\right)\right)$, we conclude that

$$
-\left(\left\|y^{\prime}+\eta^{\prime}\right\|^{p-2}\left(y^{\prime}+\eta^{\prime}\right)\right)^{\prime}+\|y+\eta\|^{p-2}(y+\eta)=h
$$

and hence

$$
\left\|y^{\prime}+\eta^{\prime}\right\|^{p-2}\left(y^{\prime}+\eta^{\prime}\right) \in W^{1, q}\left(T, \mathbb{R}^{N}\right) .
$$

Let $x=y+\eta \in C^{1}\left(T, \mathbb{R}^{N}\right)$ with $\left\|x^{\prime}\right\|^{p-2} x^{\prime} \in W^{1, q}\left(T, \mathbb{R}^{N}\right)$. This is the unique solution of $(3)$.

Now let $s: \mathbb{R}^{N} \times \mathbb{R}^{N} \rightarrow C^{1}\left(T, \mathbb{R}^{N}\right)$ be the map which to each $(v, w) \in$ $\mathbb{R}^{N} \times \mathbb{R}^{N}$ assigns the unique solution $s(v, w)=x \in C^{1}\left(T, \mathbb{R}^{N}\right)$ of (3). Then let $\varrho: \mathbb{R}^{N} \times \mathbb{R}^{N} \rightarrow \mathbb{R}^{N} \times \mathbb{R}^{N}$ be defined by

$$
\varrho(v, w)=\left(-\left\|s(v, w)^{\prime}(0)\right\|^{p-2} s(v, w)^{\prime}(0),\left\|s(v, w)^{\prime}(b)\right\|^{p-2} s(v, w)^{\prime}(b)\right) .
$$

Claim 1. $\varrho$ is monotone.

Let $x=s(\alpha, \beta)$ and $x_{1}=s\left(\alpha_{1}, \beta_{1}\right)$. Using Green's identity, we have

$$
\begin{aligned}
(\varrho(\alpha, \beta) & \left.-\varrho\left(\alpha_{1}, \beta_{1}\right),\left(\begin{array}{c}
\alpha-\alpha_{1} \\
\beta-\beta_{1}
\end{array}\right)\right)_{\mathbb{R}^{2 N}} \\
= & \left(\left\|x^{\prime}(b)\right\|^{p-2} x^{\prime}(b)-\left\|x_{1}^{\prime}(b)\right\|^{p-2} x_{1}^{\prime}(b), \beta-\beta_{1}\right)_{\mathbb{R}^{N}} \\
& \quad-\left(\left\|x^{\prime}(0)\right\|^{p-2} x^{\prime}(0)-\left\|x_{1}^{\prime}(0)\right\|^{p-2} x_{1}^{\prime}(0), \alpha-\alpha_{1}\right)_{\mathbb{R}^{N}} \\
= & \int_{0}^{b}\left\|x^{\prime}(t)\right\|^{p-2}\left(x^{\prime}(t), x^{\prime}(t)-x_{1}^{\prime}(t)\right)_{\mathbb{R}^{N}} d t \\
& \quad-\int_{0}^{b}\left\|x_{1}^{\prime}(t)\right\|^{p-2}\left(x_{1}^{\prime}(t), x^{\prime}(t)-x_{1}^{\prime}(t)\right)_{\mathbb{R}^{N}} d t \\
& +\int_{0}^{b}\left(\left(\left\|x^{\prime}(t)\right\|^{p-2} x^{\prime}(t)\right)^{\prime}-\left(\left\|x_{1}^{\prime}(t)\right\|^{p-2} x_{1}^{\prime}(t)\right)^{\prime}, x(t)-x_{1}(t)\right)_{\mathbb{R}^{N}} d t .
\end{aligned}
$$

Note that

$$
\begin{aligned}
\int_{0}^{b}\left\|x^{\prime}(t)\right\|^{p-2}\left(x^{\prime}(t), x^{\prime}(t)-x_{1}^{\prime}(t)\right)_{\mathbb{R}^{N}} d t & \\
& -\int_{0}^{b}\left\|x_{1}^{\prime}(t)\right\|^{p-2}\left(x_{1}^{\prime}(t), x^{\prime}(t)-x_{1}^{\prime}(t)\right)_{\mathbb{R}^{N}} d t \geq 0
\end{aligned}
$$


(see Section 2). Also because $x=s(\alpha, \beta)$ and $x_{1}=s\left(\alpha_{1}, \beta_{1}\right)$, we have

$$
\begin{aligned}
\int_{0}^{b}\left(\left(\left\|x^{\prime}(t)\right\|^{p-2} x^{\prime}(t)\right)^{\prime}-\left(\left\|x_{1}^{\prime}(t)\right\|^{p-2} x_{1}^{\prime}(t)\right)^{\prime}, x(t)-x_{1}(t)\right)_{\mathbb{R}^{N}} d t \\
=\int_{0}^{b}\left(\|x(t)\|^{p-2} x(t)-\left\|x_{1}(t)\right\|^{p-2} x_{1}(t), x(t)-x_{1}(t)\right)_{\mathbb{R}^{N}} d t \geq 0 .
\end{aligned}
$$

Thus finally we obtain

$$
\left(\varrho(\alpha, \beta)-\varrho\left(\alpha_{1}, \beta_{1}\right),\left(\begin{array}{c}
\alpha-\alpha_{1} \\
\beta-\beta_{1}
\end{array}\right)\right)_{\mathbb{R}^{2 N}} \geq 0
$$

which proves the claim.

Claim 2. $\varrho: \mathbb{R}^{N} \times \mathbb{R}^{N} \rightarrow \mathbb{R}^{N} \times \mathbb{R}^{N}$ is continuous.

Assume that $\alpha_{n} \rightarrow \alpha$ and $\beta_{n} \rightarrow \beta$ in $\mathbb{R}^{N}$ and set $x_{n}=s\left(\alpha_{n}, \beta_{n}\right), n \geq 1$, $x=s(\alpha, \beta)$. As before we introduce $\eta_{n}(t)=(1-t / b) \alpha_{n}+(t / b) \beta_{n}, n \geq 1$, and $\eta(t)=(1-t / b) \alpha+(t / b) \beta$ and set $y_{n}=x_{n}-\eta_{n}$. We have

$$
\begin{aligned}
-\int_{0}^{b}\left(\left(\| y_{n}^{\prime}+\right.\right. & \left.\left.\eta_{n}^{\prime} \|^{p-2}\left(y_{n}^{\prime}+\eta_{n}^{\prime}\right)\right)^{\prime}, y_{n}\right)_{\mathbb{R}^{N}} d t \\
& +\int_{0}^{b}\left\|y_{n}+\eta_{n}\right\|^{p-2}\left(y_{n}+\eta_{n}, y_{n}\right)_{\mathbb{R}^{N}} d t=\int_{0}^{b}\left(h(t), y_{n}(t)\right)_{\mathbb{R}^{N}} d t
\end{aligned}
$$

that is,

$$
\begin{array}{r}
\int_{0}^{b}\left\|y_{n}^{\prime}+\eta_{n}^{\prime}\right\|^{p-2}\left(y_{n}^{\prime}+\eta_{n}^{\prime}, y_{n}^{\prime}\right)_{\mathbb{R}^{N}} d t+\int_{0}^{b}\left\|y_{n}+\eta_{n}\right\|^{p-2}\left(y_{n}+\eta_{n}, y_{n}\right)_{\mathbb{R}^{N}} d t \\
=\int_{0}^{b}\left(h(t), y_{n}(t)\right)_{\mathbb{R}^{N}} d t \quad \text { (by Green's identity), }
\end{array}
$$

therefore

$$
\begin{aligned}
\left\|y_{n}^{\prime}+\eta_{n}^{\prime}\right\|_{p}^{p}-k_{3}\left\|y_{n}^{\prime}+\eta_{n}^{\prime}\right\|_{p}^{p-1}+\left\|y_{n}+\eta_{n}\right\|_{p}^{p}-k_{4}\left\|y_{n}+\eta_{n}\right\|_{p}^{p-1} & \leq\|h\|_{q}\left\|y_{n}+\eta_{n}\right\|_{p}+k_{5}
\end{aligned}
$$

for some $k_{3}, k_{4}, k_{5}>0$, and so $\left\{x_{n}=y_{n}+\eta_{n}\right\}_{n \geq 1} \subseteq W^{1, p}\left(T, \mathbb{R}^{N}\right)$ is bounded. Thus $\left\{\left\|x_{n}\right\|^{p-2} x_{n}\right\}_{n \geq 1},\left\{\left\|x_{n}^{\prime}\right\|^{p-2} x_{n}^{\prime}\right\}_{n \geq 1} \subseteq L^{q}\left(T, \mathbb{R}^{N}\right)$ are both bounded. Moreover, from (3) it follows that $\left\{\left\|x_{n}^{\prime}\right\|^{p-2} x_{n}^{\prime}\right\}_{n \geq 1} \subseteq W^{1, q}\left(T, \mathbb{R}^{N}\right)$ is bounded. So we may assume that $x_{n} \stackrel{w}{\rightarrow} u$ in $W^{1, p}\left(T, \mathbb{R}^{N}\right)$ and $\left\|x_{n}^{\prime}\right\|^{p-2} x_{n}^{\prime}$ $\stackrel{w}{\rightarrow} v$ in $W^{1, q}\left(T, \mathbb{R}^{N}\right)$ as $n \rightarrow \infty$. In particular $x_{n} \rightarrow u$ in $C\left(T, \mathbb{R}^{N}\right)$ (recall that $W^{1, p}\left(T, \mathbb{R}^{N}\right)$ is compactly embedded in $\left.C\left(T, \mathbb{R}^{N}\right)\right)$ and $\left(\left\|x_{n}^{\prime}\right\|^{p-2} x_{n}^{\prime}\right)^{\prime}$ 
$\stackrel{w}{\rightarrow} v^{\prime}$ in $L^{q}\left(T, \mathbb{R}^{N}\right)$ as $n \rightarrow \infty$. Thus in the limit as $n \rightarrow \infty$, we obtain

$$
\left\{\begin{array}{l}
-v^{\prime}(t)+\|u(t)\|^{p-2} u(t)=h(t) \quad \text { a.e. on } T, \\
u(0)=\alpha, \quad u(b)=\beta .
\end{array}\right.
$$

Since $\left\|x_{n}^{\prime}\right\|^{p-2} x_{n}^{\prime} \stackrel{w}{\rightarrow} v$ in $W^{1, q}\left(T, \mathbb{R}^{N}\right)$, we have $\left\|x_{n}^{\prime}\right\|^{p-2} x_{n}^{\prime} \rightarrow v$ in $C\left(T, \mathbb{R}^{N}\right)$ (from the compact embedding of $W^{1, q}\left(T, \mathbb{R}^{N}\right)$ in $C\left(T, \mathbb{R}^{N}\right)$ ). Also $\varphi^{-1}$ exists and is continuous because $\varphi(r)=\|r\|^{p-2} r$ is a homeomorphism. Therefore $\varphi^{-1}\left(\left\|x_{n}^{\prime}\right\|^{p-2} x_{n}^{\prime}\right)=x_{n}^{\prime} \rightarrow \varphi^{-1}(v)$ in $L^{p}\left(T, \mathbb{R}^{N}\right)$ (in fact in $\left.C\left(T, \mathbb{R}^{N}\right)\right)$, hence $\varphi^{-1}(v)=u^{\prime}$ and so $v=\varphi\left(u^{\prime}\right)=\left\|u^{\prime}\right\|^{p-2} u^{\prime}$. Thus finally we have

$$
\left\{\begin{array}{l}
-\left(\left\|u^{\prime}(t)\right\|^{p-2} u^{\prime}(t)\right)^{\prime}+\|u(t)\|^{p-2} u(t)=h(t) \quad \text { a.e. on } T \\
u(0)=\alpha, \quad u(b)=\beta .
\end{array}\right.
$$

Hence $u=s(\alpha, \beta)=x$, i.e. $s$ is continuous from $\mathbb{R}^{N} \times \mathbb{R}^{N}$ into $C^{1}\left(T, \mathbb{R}^{N}\right)$.

From the continuity of $s$ we deduce at once the continuity of $\varrho$.

Claim 3. @ is coercive.

We have, with $x=s(\alpha, \beta)$,

$$
\begin{aligned}
\frac{\left(\varrho(\alpha, \beta),\left(\begin{array}{l}
\alpha \\
\beta
\end{array}\right)\right)_{\mathbb{R}^{2 N}}}{\left\|\left(\begin{array}{c}
\alpha \\
\beta
\end{array}\right)\right\|} & =\frac{\left\|x^{\prime}(b)\right\|^{p-2}\left(x^{\prime}(b), \beta\right)_{\mathbb{R}^{N}}-\left\|x^{\prime}(0)\right\|^{p-2}\left(x^{\prime}(0), \alpha\right)_{\mathbb{R}^{N}}}{\left\|\left(\begin{array}{c}
\alpha \\
\beta
\end{array}\right)\right\|} \\
& =\frac{\int_{0}^{b}\left(\left(\left\|x^{\prime}(t)\right\|^{p-2} x^{\prime}(t)\right)^{\prime}, x(t)\right)_{\mathbb{R}^{N}} d t+\left\|x^{\prime}\right\|_{p}^{p}}{\left\|\left(\begin{array}{c}
\alpha \\
\beta
\end{array}\right)\right\|} \\
& \geq \frac{\|x\|_{p}^{p}+\left\|x^{\prime}\right\|_{p}^{p}-\|h\|_{q}\|x\|_{p}}{\left\|\left(\begin{array}{c}
\alpha \\
\beta
\end{array}\right)\right\|}
\end{aligned}
$$

where we have used Green's identity and (3).

From the mean value theorem for integrals (see for example HewittStromberg [9], Theorem 21.69, p. 420), we can find $t_{0} \in T$ such that $\left\|x\left(t_{0}\right)\right\| b=\int_{0}^{b}\|x(t)\| d t$. So for every $t \in T$,

$$
\|x(t)\| \leq\left\|x\left(t_{0}\right)\right\|+\int_{t_{0}}^{t}\left\|x^{\prime}(s)\right\| d s \leq \frac{1}{b}\|x\|_{1}+b^{1 / q}\left\|x^{\prime}\right\|_{p} \leq k_{6}\|x\|_{1, p}
$$

for some $k_{6}>0$ and hence $\left\|\left(\begin{array}{l}\alpha \\ \beta\end{array}\right)\right\| \leq k_{7}\|x\|_{1, p}$ for some $k_{7}>0$. Therefore we have

i.e. $\varrho$ is coercive.

$$
\frac{\left(\varrho(\alpha, \beta),\left(\left(\begin{array}{c}
\alpha \\
\beta
\end{array}\right)\right)_{\mathbb{R}^{2 N}}\right.}{\left\|\left(\begin{array}{c}
\alpha \\
\beta
\end{array}\right)\right\|} \geq \frac{\|x\|_{1, p}^{p}-\|h\|_{q}\|x\|_{1, p}}{k_{7}\|x\|_{1, p}},
$$

From Claims 1, 2 and 3, it follows that $\varrho$ is maximal monotone.

Now let $\sigma=\varrho+\xi: \mathbb{R}^{N} \times \mathbb{R}^{N} \rightarrow 2^{\mathbb{R}^{N} \times \mathbb{R}^{N}}$. Then $\sigma$ is maximal monotone (see Hu-Papageorgiou [10], Theorem III.3.3, p. 334) and coercive (recall 
that $\varrho$ is coercive by Claim 3 and $(0,0) \in \xi(0,0)$ by hypothesis $\mathrm{H}(\xi))$. Thus $\sigma$ is surjective. So we can find $(\alpha, \beta) \in \mathbb{R}^{N} \times \mathbb{R}^{N}$ such that $0 \in \sigma(\alpha, \beta)$. Let $x=s(\alpha, \beta)$. Evidently this is the desired solution of $(2)$.

$$
\begin{aligned}
& \text { Let } \\
& \begin{array}{r}
D=\left\{x \in C^{1}\left(T, \mathbb{R}^{N}\right):\left\|x^{\prime}(\cdot)\right\|^{p-2} x^{\prime}(\cdot) \in W^{1, q}\left(T, \mathbb{R}^{N}\right),\right. \\
\left.\left(\varphi\left(x^{\prime}(0)\right),-\varphi\left(x^{\prime}(b)\right)\right) \in \xi(x(0), x(b))\right\}
\end{array}
\end{aligned}
$$

and let $V: D \subseteq L^{p}\left(T, \mathbb{R}^{N}\right) \rightarrow L^{q}\left(T, \mathbb{R}^{N}\right)$ be defined by

$$
V(x)(\cdot)=-\left(\left\|x^{\prime}(\cdot)\right\|^{p-2} x^{\prime}(\cdot)\right)^{\prime}, \quad x \in D .
$$

Proposition 3. If $\xi: \mathbb{R}^{N} \times \mathbb{R}^{N} \rightarrow 2^{\mathbb{R}^{N} \times \mathbb{R}^{N}}$ is a maximal monotone map with $(0,0) \in \xi(0,0)$, then $V: D \subseteq L^{p}\left(T, \mathbb{R}^{N}\right) \rightarrow L^{q}\left(T, \mathbb{R}^{N}\right)$ is maximal monotone.

Pr o of. First note that for every $x, y \in D$, we have

$$
(V(x)-V(y), x-y)_{p q} \geq \int_{0}^{b}\left(\left\|x^{\prime}(t)\right\|^{p-1}-\left\|y^{\prime}(t)\right\|^{p-1}\right)\left(\left\|x^{\prime}(t)\right\|-\left\|y^{\prime}(t)\right\|\right) d t \geq 0,
$$

hence $V$ is monotone.

To prove the maximality of $V$ it suffices to show $R(V+J)=L^{q}\left(T, \mathbb{R}^{N}\right)$ with $J: L^{p}\left(T, \mathbb{R}^{N}\right) \rightarrow L^{q}\left(T, \mathbb{R}^{N}\right)$ defined by $J(x)(\cdot)=\|x(\cdot)\|^{p-2} x(\cdot)$. Indeed, assume for the moment that $V+J$ is surjective and suppose that $y \in L^{p}\left(T, \mathbb{R}^{N}\right)$ and $v \in L^{q}\left(T, \mathbb{R}^{N}\right)$ satisfy

$$
(V(x)-v, x-y)_{p q} \geq 0 \quad \text { for all } x \in D .
$$

Let $x_{1} \in D$ be such that $v+J(y)=V\left(x_{1}\right)+J\left(x_{1}\right)$. Thus we can write

$$
0 \leq\left(V\left(x_{1}\right)-V\left(x_{1}\right)-J\left(x_{1}\right)+J(y), x_{1}-y\right)_{p q}=\left(J(y)-J\left(x_{1}\right), x_{1}-y\right)_{p q} .
$$

But it is easy to see that $J$ is strictly monotone. So from this last inequality it follows that $y=x_{1} \in D$ and $v=V\left(x_{1}\right)$, which proves the maximality of $V$. Therefore we need to show that $R(V+J)=L^{q}\left(T, \mathbb{R}^{N}\right)$. But this is an immediate consequence of Proposition 2.

Next let $\lambda>0$ and let $A_{\lambda}: \mathbb{R}^{N} \rightarrow \mathbb{R}^{N}$ be the Yosida approximation of $A$. Note that as indicated in the remark following hypothesis $\mathrm{H}(A)$, we may assume without loss of generality that $0 \in A(0)$, which in turn implies that $0=A_{\lambda}(0)$ for all $\lambda>0$. Let $\widehat{A}_{\lambda}: L^{p}\left(T, \mathbb{R}^{N}\right) \rightarrow L^{q}\left(T, \mathbb{R}^{N}\right)$ be the Nemytskiŭ operator corresponding to $A_{\lambda}$, i.e. $\widehat{A}_{\lambda}(x)(\cdot)=A_{\lambda}(x(\cdot))$ for all $x \in$ $L^{p}\left(T, \mathbb{R}^{N}\right)$. Clearly $\widehat{A}_{\lambda}$ is monotone, continuous (Krasnosel'skiı̌'s theorem), thus maximal monotone. We consider the following auxiliary boundary value problem:

$$
\left\{\begin{array}{l}
\left(\left\|x^{\prime}(t)\right\|^{p-2} x^{\prime}(t)\right)^{\prime}=A_{\lambda}(x(t))+f\left(t, x(t), x^{\prime}(t)\right) \quad \text { a.e. on } T \\
\left(\varphi\left(x^{\prime}(0)\right),-\varphi\left(x^{\prime}(b)\right)\right) \in \xi(x(0), x(b)) .
\end{array}\right.
$$


Proposition 4. If hypotheses $\mathrm{H}(A)_{2}, \mathrm{H}(f)_{1}$ (or $\left.\mathrm{H}(f)_{2}\right)$ and $\mathrm{H}(\xi)$ hold, then problem (7) has a solution $x \in C^{1}\left(T, \mathbb{R}^{N}\right)$.

Pr o of. We do the proof when $\mathrm{H}(f)_{1}$ holds, the other case being similar. Let $K_{\lambda}=V+\widehat{A}_{\lambda}+J: D \subseteq L^{p}\left(T, \mathbb{R}^{N}\right) \rightarrow L^{q}\left(T, \mathbb{R}^{N}\right), \lambda>0$. Note that $K_{\lambda}$ is maximal monotone (see Proposition 3 and Theorem III.3.3, p. 334, of $\mathrm{Hu}$-Papageorgiou [10]). Also since $0=A_{\lambda}(0)$, we have

$$
\left(K_{\lambda}(x), x\right)_{p q} \geq(V(x), x)_{p q}+(J(x), x)_{p q} .
$$

But

$$
\begin{aligned}
(V(x), x)_{p q}= & -\int_{0}^{b}\left(\left(\left\|x^{\prime}(t)\right\|^{p-2} x^{\prime}(t)\right)^{\prime}, x(t)\right)_{\mathbb{R}^{N}} d t \\
= & \left(-\left\|x^{\prime}(b)\right\|^{p-2} x^{\prime}(b), x(b)\right)_{\mathbb{R}^{N}} \\
& +\left(\left\|x^{\prime}(0)\right\|^{p-2} x^{\prime}(0), x(0)\right)_{\mathbb{R}^{N}}+\left\|x^{\prime}\right\|_{p}^{p} \\
\geq & \left\|x^{\prime}\right\|_{p}^{p} .
\end{aligned}
$$

Here we have used Green's identity and the fact that $\left(\varphi\left(x^{\prime}(0)\right),-\varphi\left(x^{\prime}(b)\right)\right) \in$ $\xi(x(0), x(b))$ and $(0,0) \in \xi(0,0)$. Also $(J(x), x)_{p q}=\|x\|_{p}^{p}$. Thus finally

$$
\left(K_{\lambda}(x), x\right)_{p q} \geq\left\|x^{\prime}\right\|_{p}^{p}+\|x\|_{p}^{p}=\|x\|_{1, p}^{p},
$$

thus $K_{\lambda}$ is coercive, hence surjective. Moreover, from the strict monotonicity of $J$, it follows that $K_{\lambda}$ is injective. So $K_{\lambda}^{-1}: L^{q}\left(T, \mathbb{R}^{N}\right) \rightarrow D \subseteq W^{1, p}\left(T, \mathbb{R}^{N}\right)$ is well defined.

Claim 1. $K_{\lambda}^{-1}$ is compact from $L^{q}\left(T, \mathbb{R}^{N}\right)$ into $W^{1, p}\left(T, \mathbb{R}^{N}\right)$.

By the reflexivity of $L^{q}\left(T, \mathbb{R}^{N}\right)$, to establish the claim, it suffices to show that if $u_{n} \stackrel{w}{\rightarrow} u$ in $L^{q}\left(T, \mathbb{R}^{N}\right)$, then $K_{\lambda}^{-1}\left(u_{n}\right) \rightarrow K_{\lambda}^{-1}(u)$ in $W^{1, p}\left(T, \mathbb{R}^{N}\right)$. Let $x_{n}=K_{\lambda}^{-1}\left(u_{n}\right), n \geq 1$. We have

$$
\left(V\left(x_{n}\right), x_{n}\right)_{p q}+\left(\widehat{A}_{\lambda}\left(x_{n}\right), x_{n}\right)_{p q}+\left(J\left(x_{n}\right), x_{n}\right)_{p q}=\left(u_{n}, x_{n}\right)_{p q},
$$

hence $\left\|x_{n}^{\prime}\right\|_{p}^{p}+\left\|x_{n}\right\|_{p}^{p} \leq\left\|u_{n}\right\|_{q}\left\|x_{n}\right\|_{p}$, and thus $\left\{x_{n}\right\}_{n \geq 1} \subseteq W^{1, p}\left(T, \mathbb{R}^{N}\right)$ is bounded. Thus we may assume that $x_{n} \stackrel{w}{\rightarrow} x$ in $W^{1, p}\left(T, \mathbb{R}^{N}\right)$ and $x_{n} \rightarrow x$ in $L^{p}\left(T, \mathbb{R}^{N}\right)$ (because $W^{1, p}\left(T, \mathbb{R}^{N}\right)$ is compactly embedded in $L^{p}\left(T, \mathbb{R}^{N}\right)$ ). Since $\left(x_{n}, u_{n}\right) \in \operatorname{Gr} K_{\lambda}, n \geq 1$, and $K_{\lambda}$ is maximal monotone (it has a demiclosed graph in $L^{p}\left(T, \mathbb{R}^{N}\right) \times L^{q}\left(T, \mathbb{R}^{N}\right)$, see Section 2), we have $(x, u) \in$ $\operatorname{Gr} K_{\lambda}$. So $u=V(x)+\widehat{A}_{\lambda}(x)+J(x)$. Moreover

$$
\lim \left(V\left(x_{n}\right)+\widehat{A}_{\lambda}\left(x_{n}\right)+J\left(x_{n}\right), x_{n}-x\right)_{p q}=\lim \left(u_{n}, x_{n}-x\right)_{p q}=0
$$

and hence $\lim \left(V\left(x_{n}\right), x_{n}-x\right)_{p q}=0\left(\right.$ since $\left(\widehat{A}_{\lambda}\left(x_{n}\right), x_{n}-x\right)_{p q} \rightarrow 0$ and $\left.\left(J\left(x_{n}\right), x_{n}-x\right)_{p q} \rightarrow 0\right)$.

But, by Proposition 3, we know that $V$ is maximal monotone, hence it is also generalized pseudomonotone (see $\mathrm{Hu}$-Papageorgiou [10], Definition 
III.6.2 and Remark III.6.3, p. 365). Therefore $V\left(x_{n}\right) \stackrel{w}{\rightarrow} V(x)$ in $L^{q}\left(T, \mathbb{R}^{N}\right)$. Note that $\left\{\left\|x_{n}^{\prime}\right\|^{p-2} x_{n}^{\prime}\right\}_{n \geq 1} \subseteq W^{1, q}\left(T, \mathbb{R}^{N}\right)$ and

$$
\left\|x_{n}^{\prime}\right\|^{p-2} x_{n}^{\prime} \stackrel{w}{\rightarrow}\left\|x^{\prime}\right\|^{p-2} x^{\prime} \quad \text { in } W^{1, q}\left(T, \mathbb{R}^{N}\right)
$$

hence

$$
\left\|x_{n}^{\prime}\right\|^{p-2} x_{n}^{\prime} \rightarrow\left\|x^{\prime}\right\|^{p-2} x^{\prime} \quad \text { in } C\left(T, \mathbb{R}^{N}\right) .
$$

So acting with $\varphi^{-1}$ we obtain $x_{n}^{\prime} \rightarrow x^{\prime}$ in $L^{p}\left(T, \mathbb{R}^{N}\right)$ as $n \rightarrow \infty$. Since $x_{n} \rightarrow x$ in $L^{p}\left(T, \mathbb{R}^{N}\right)$, we conclude that $x_{n} \rightarrow x$ in $W^{1, p}\left(T, \mathbb{R}^{N}\right)$ and this proves the claim.

Let $N: W^{1, p}\left(T, \mathbb{R}^{N}\right) \rightarrow L^{q}\left(T, \mathbb{R}^{N}\right)$ be the Nemytskiü operator corresponding to $f$, i.e. $N(x)(\cdot)=f\left(\cdot, x(\cdot), x^{\prime}(\cdot)\right)$. Clearly $N$ is continuous (see hypothesis $\mathrm{H}(f))$. Let $N_{1}=-N+J$ and consider the following abstract fixed point problem:

$$
x=K_{\lambda}^{-1} N_{1}(x) .
$$

Observe that $N_{1}: W^{1, p}\left(T, \mathbb{R}^{N}\right) \rightarrow L^{q}\left(T, \mathbb{R}^{N}\right)$ is continuous, bounded and $K_{\lambda}^{-1}: L^{q}\left(T, \mathbb{R}^{N}\right) \rightarrow W^{1, p}\left(T, \mathbb{R}^{N}\right)$ is compact. Hence $K_{\lambda}^{-1} N_{1}: W^{1, p}\left(T, \mathbb{R}^{N}\right)$ $\rightarrow W^{1, p}\left(T, \mathbb{R}^{N}\right)$ is compact. So according to Theorem 1 , in order to solve (8), it suffices to prove the following claim:

Claim 2. $S=\left\{x \in W^{1, p}\left(T, \mathbb{R}^{N}\right): x=\beta K_{\lambda}^{-1} N_{1}(x), 0<\beta<1\right\}$ is bounded.

Let $x \in S$. We have $K_{\lambda}((1 / \beta) x)=N_{1}(x)$ and so

$$
V\left(\frac{1}{\beta} x\right)+\widehat{A}_{\lambda}\left(\frac{1}{\beta} x\right)+J\left(\frac{1}{\beta} x\right)=-N(x)+J(x) .
$$

Hence

$$
\begin{aligned}
\left(V\left(\frac{1}{\beta} x\right), x\right)_{p q}+\left(\widehat{A}_{\lambda}\left(\frac{1}{\beta} x\right), x\right)_{p q} & \left(J\left(\frac{1}{\beta} x\right), x\right)_{p q} \\
& =-(N(x), x)_{p q}+(J(x), x)_{p q} .
\end{aligned}
$$

Note that $\left(\widehat{A}_{\lambda}((1 / \beta) x), x\right)_{p q} \geq 0$ (recall that $0=\widehat{A}_{\lambda}(0)$ and $\widehat{A}_{\lambda}$ is monotone). Moreover,

$$
\begin{aligned}
\left(V\left(\frac{1}{\beta} x\right), x\right)_{p q}= & -\int_{0}^{b} \frac{1}{\beta^{p-1}}\left(\left(\left\|x^{\prime}(t)\right\|^{p-2} x^{\prime}(t)\right)^{\prime}, x(t)\right)_{\mathbb{R}^{N}} d t \\
= & -\left(\frac{1}{\beta^{p-1}}\left\|x^{\prime}(b)\right\|^{p-2} x^{\prime}(b), x(b)\right)_{\mathbb{R}^{N}} \\
& +\left(\frac{1}{\beta^{p-1}}\left\|x^{\prime}(0)\right\|^{p-2} x^{\prime}(0), x(0)\right)_{\mathbb{R}^{N}}+\frac{1}{\beta^{p-1}}\left\|x^{\prime}\right\|_{p}^{p} \\
\geq & \frac{1}{\beta^{p-1}}\left\|x^{\prime}\right\|_{p}^{p} \quad \text { (from the boundary conditions). }
\end{aligned}
$$


So we have

$$
\frac{1}{\beta^{p-1}}\left\|x^{\prime}\right\|_{p}^{p}+\frac{1}{\beta^{p-1}}\|x\|_{p}^{p} \leq-(N(x), x)_{p q}+\|x\|_{p}^{p}
$$

and hence

$$
\left\|x^{\prime}\right\|_{p}^{p} \leq-\beta^{p-1}(N(x), x)_{p q}+\left(\beta^{p-1}-1\right)\|x\|_{p}^{p} \leq-\beta^{p-1}(N(x), x)_{p q}
$$

(since $0<\beta<1$ ). Using hypothesis $\mathrm{H}(f)$ (iii), we have

$$
\begin{aligned}
-\beta^{p-1}(N(x), x)_{p q}= & \beta^{p-1} \int_{0}^{b}-\left(f\left(t, x(t), x^{\prime}(t)\right), x(t)\right)_{\mathbb{R}^{N}} d t \\
\leq & \beta^{p-1} a\|x\|_{p}^{p}+\beta^{p-1} \gamma \int_{0}^{b}\|x(t)\|^{r}\left\|x^{\prime}(t)\right\|^{p-r} d t \\
& +\beta^{p-1}\|c\|_{1}\|x\|_{\infty}^{s} .
\end{aligned}
$$

Let $\tau=p-r$ and set $\mu=p / r, \mu^{\prime}=p / \tau\left(1 / \mu+1 / \mu^{\prime}=1\right)$. Apply Hölder's inequality to obtain

$$
\begin{aligned}
\int_{0}^{b}\|x(t)\|^{r}\left\|x^{\prime}(t)\right\|^{p-r} d t & \leq\left(\int_{0}^{b}\|x(t)\|^{r \mu} d t\right)^{1 / \mu}\left(\int_{0}^{b}\left\|x^{\prime}(t)\right\|^{\tau \mu^{\prime}} d t\right)^{1 / \mu^{\prime}} \\
& \leq\|x\|_{p}^{r}\left\|x^{\prime}\right\|_{p}^{\tau} .
\end{aligned}
$$

It follows that

(10) $\quad-\beta^{p-1}(N(x), x)_{p q} \leq \beta^{p-1} a\|x\|_{p}^{p}+\beta^{p-1} \gamma\|x\|_{p}^{r}\left\|x^{\prime}\right\|_{p}^{\tau}+\beta^{p-1}\|c\|_{1}\|x\|_{\infty}^{s}$.

Next we will show that for all $x \in S$ we have $\|x\|_{\infty} \leq M$ (with $M>0$ as in hypothesis $\mathrm{H}(f)(\mathrm{iv}))$. To this end let $r(t)=\|x(t)\|^{p}$ and let $t_{0} \in T$ be the point where $r(\cdot)$ attains its maximum. Suppose that $r\left(t_{0}\right)>M^{p}$ and assume first that $0<t_{0}<b$. Then $0=r^{\prime}\left(t_{0}\right)=p\left\|x\left(t_{0}\right)\right\|^{p-2}\left(x^{\prime}\left(t_{0}\right), x\left(t_{0}\right)\right)_{\mathbb{R}^{N}}$, hence $\left(x^{\prime}\left(t_{0}\right), x\left(t_{0}\right)\right)_{\mathbb{R}^{N}}=0$. By hypothesis $\mathrm{H}(f)(\mathrm{iv})$, we can find $\delta, \xi>0$ such that

$$
\inf \left[(f(t, x, y), x)_{\mathbb{R}^{N}}+\|y\|^{p}:\left\|x-x\left(t_{0}\right)\right\|+\left\|y-x^{\prime}\left(t_{0}\right)\right\|<\delta\right] \geq \xi>0 .
$$

Note that since $x \in S$ we see that $\left\|x^{\prime}(\cdot)\right\|^{p-2} x^{\prime}(\cdot) \in W^{1, q}\left(T, \mathbb{R}^{N}\right) \subseteq$ $C\left(T, \mathbb{R}^{N}\right)$, hence $\varphi^{-1}\left(\left\|x^{\prime}(\cdot)\right\|^{p-2} x^{\prime}(\cdot)\right)=x^{\prime}(\cdot) \in C\left(T, \mathbb{R}^{N}\right)$. Thus we can find $\delta_{1}>0$ such that if $t_{0}<t \leq t_{0}+\delta_{1}$, then

$$
\left\|x(t)-x\left(t_{0}\right)\right\|+\left\|x^{\prime}(t)-x^{\prime}\left(t_{0}\right)\right\|<\delta
$$

Therefore for almost all $t \in\left(t_{0}, t_{0}+\delta_{1}\right]$ we have

$$
\beta^{p-1}\left(f\left(t, x(t), x^{\prime}(t)\right), x(t)\right)_{\mathbb{R}^{N}}+\beta^{p-1}\left\|x^{\prime}(t)\right\|^{p} \geq \beta^{p-1} \xi .
$$

Now from the equation $V\left(\beta^{-1} x\right)+\widehat{A}_{\lambda}\left(\beta^{-1} x\right)+J\left(\beta^{-1} x\right)=-N(x)+J(x)$ it follows that 


$$
\begin{aligned}
& -\left(\left\|x^{\prime}(t)\right\|^{p-2} x^{\prime}(t)\right)^{\prime}+\beta^{p-1} A_{\lambda} \beta^{-1} x(t) \\
& \quad=-\beta^{p-1} f\left(t, x(t), x^{\prime}(t)\right)\left(\beta^{p-1}-1\right)\|x(t)\|^{p-2} x(t) \quad \text { a.e. on } T .
\end{aligned}
$$

Using this last equality in (11), we have

$$
\begin{aligned}
\left(\left(\left\|x^{\prime}(t)\right\|^{p-2} x^{\prime}(t)\right)^{\prime}, x(t)\right)_{\mathbb{R}^{N}}-\beta^{p-1}\left(A_{\lambda}\left(\beta^{-1} x(t)\right), x(t)\right)_{\mathbb{R}^{N}} \\
+\left(\beta^{p-1}-1\right)\|x(t)\|^{p}+\beta^{p-1}\left\|x^{\prime}(t)\right\|^{p} \geq \beta^{p-1} \xi
\end{aligned}
$$

and hence

$$
\int_{t_{0}}^{t}\left(\left(\left\|x^{\prime}(s)\right\|^{p-2} x^{\prime}(s)\right)^{\prime}, x(s)\right)_{\mathbb{R}^{N}} d s+\beta^{p-1} \int_{t_{0}}^{t}\left\|x^{\prime}(s)\right\|^{p} d s \geq \beta^{p-1} \xi\left(t-t_{0}\right) .
$$

Using Green's identity for the first integral and the fact that $\left(x^{\prime}\left(t_{0}\right), x\left(t_{0}\right)\right)_{\mathbb{R}^{N}}$ $=0$, we have

$$
\begin{aligned}
\left\|x^{\prime}(t)\right\|^{p-2}\left(x^{\prime}(t), x(t)\right)_{\mathbb{R}^{N}}-\int_{t_{0}}^{t}\left\|x^{\prime}(s)\right\|^{p} d s+\beta^{p-1} \int_{t_{0}}^{t}\left\|x^{\prime}(s)\right\|^{p} d s & \\
& \geq \beta^{p-1} \xi\left(t-t_{0}\right) .
\end{aligned}
$$

Therefore $\left\|x^{\prime}(t)\right\|^{p-2}\left(x^{\prime}(t), x(t)\right)_{\mathbb{R}^{N}} \geq \beta^{p-1} \xi\left(t-t_{0}\right.$ ) (since $0<\beta<1$ ) and so $r^{\prime}(t)>0$ for all $t \in\left(t_{0}, t_{0}+\delta_{1}\right]$. Thus $r(t)>r\left(t_{0}\right)$ for $t_{0}<t \leq t_{0}+\delta_{1}$, a contradiction to the choice of $t_{0}$. So $\|x(t)\| \leq M$ for all $t \in T$ and all $x \in S$, provided $t_{0} \in(0, b)$.

Next let $t_{0}=0$. Then $r^{\prime}(0) \leq 0$ and so $\|x(0)\|^{p-2}\left(x^{\prime}(0), x(0)\right)_{\mathbb{R}^{N}} \leq 0$, hence $\left(x^{\prime}(0), x(0)\right)_{\mathbb{R}^{N}} \leq 0$. First suppose that hypothesis $\mathrm{H}(\xi)(\mathrm{i})$ is in effect. We have $\left\|x^{\prime}(0)\right\|^{p-2}\left(x^{\prime}(0), x(0)\right)_{\mathbb{R}^{N}} \geq 0$, hence $\left(x^{\prime}(0), x(0)\right)_{\mathbb{R}^{N}} \geq 0$ and finally $\left(x^{\prime}(0), x(0)\right)_{\mathbb{R}^{N}}=0$ and so we can proceed as before. Now suppose that hypothesis $\mathrm{H}(\xi)(\mathrm{ii})$ is in effect. We may assume that $x \neq 0$. Then $x(0)=x(b)$ and $r^{\prime}(0) \leq 0 \leq r^{\prime}(b)$, which implies that

$$
\|x(0)\|^{p-2}\left(x^{\prime}(0), x(0)\right)_{\mathbb{R}^{N}} \leq 0 \leq\|x(b)\|^{p-2}\left(x^{\prime}(b), x(b)\right)_{\mathbb{R}^{N}}
$$

and so

$$
\left(x^{\prime}(0), x(0)\right)_{\mathbb{R}^{N}} \leq 0 \leq\left(x^{\prime}(b), x(b)\right)_{\mathbb{R}^{N}} .
$$

Also from the boundary conditions, we see that $\left\|x^{\prime}(b)\right\|^{p-2}\left(x^{\prime}(b), x(b)\right)_{\mathbb{R}^{N}} \leq$ $\left\|x^{\prime}(0)\right\|^{p-2}\left(x^{\prime}(0), x(0)\right)_{\mathbb{R}^{N}}$. Thus we obtain either $x^{\prime}(0)=x^{\prime}(b)=0$, from which it follows that $r^{\prime}(0)=r^{\prime}(b)=0$, or $\left(x^{\prime}(0), x(0)\right)_{\mathbb{R}^{N}}=\left(x^{\prime}(b), x(b)\right)_{\mathbb{R}^{N}}$, from which again it follows that $r^{\prime}(0)=r^{\prime}(b)=0$. So we always have $r^{\prime}(0)=0$ and proceed as before. Similarly we treat the case $t_{0}=b$. Hence we have established that $\|x\|_{\infty} \leq M$ for all $x \in S$. Then from (10) we have

$$
\beta^{p-1}(-N(x), x)_{p q} \leq k_{8}+k_{9}\left\|x^{\prime}\right\|_{p}^{\tau} \quad \text { for some } k_{8}, k_{9}>0 .
$$

Using this estimate in (9), we obtain

$$
\left\|x^{\prime}\right\|_{p}^{p} \leq k_{8}+k_{9}\left\|x^{\prime}\right\|_{p}^{\tau} \quad(\tau<p)
$$


which yields that $\left\{x^{\prime}\right\}_{s \in S} \subseteq L^{p}\left(T, \mathbb{R}^{N}\right)$ is bounded, and therefore $\{x\}_{s \in S} \subseteq$ $W^{1, p}\left(T, \mathbb{R}^{N}\right)$ is bounded. This proves Claim 2 .

Now we can apply Theorem 1 to obtain $x \in D$ such that $x=K_{\lambda}^{-1} N_{1}(x)$, that is, $K_{\lambda}(x)=N_{1}(x)$ and thus $x$ is a solution of $(7)$.

4. Main results, special cases. In this section we state and prove the main existence theorems concerning problem (1) and present some special cases of interest, which illustrate the generality of our results.

TheOREM 5. If hypotheses $\mathrm{H}(A)_{1}, \mathrm{H}(f)_{1}$ and $\mathrm{H}(\xi)$ hold, then problem (1) has a solution $x \in C^{1}\left(T, \mathbb{R}^{N}\right)$.

Proof. Let $\lambda_{n} \rightarrow 0, \lambda_{n}>0$ and let $x_{n} \in C^{1}\left(T, \mathbb{R}^{N}\right)$ be solutions of the auxiliary problem (7). As in the proof of Proposition 4, we can show that $\left\|x_{n}(t)\right\| \leq M$ for all $t \in T$ and all $n \geq 1$. Also we have $V\left(x_{n}\right)+\widehat{A}_{\lambda_{n}}\left(x_{n}\right)=$ $-N\left(x_{n}\right)$, which yields

$$
\left(V\left(x_{n}\right), x_{n}\right)_{p q}+\left(\widehat{A}_{\lambda_{n}}\left(x_{n}\right), x_{n}\right)_{p q}=-\left(N\left(x_{n}\right), x_{n}\right)_{p q}
$$

and therefore

$$
\left\|x_{n}^{\prime}\right\|_{p}^{p} \leq\left\|N\left(x_{n}\right)\right\|_{q}\left\|x_{n}\right\|_{p} \leq k_{10}\left\|N\left(x_{n}\right)\right\|_{q} \quad \text { for some } k_{10}>0 .
$$

Here we have used Green's identity and the boundary conditions in the first term $\left(V\left(x_{n}\right), x_{n}\right)_{p q}$ and the fact that $\widehat{A}_{\lambda_{n}}(\cdot)$ is monotone with $0=\widehat{A}_{\lambda_{n}}(0)$ in the second term $\left(\widehat{A}_{\lambda_{n}}\left(x_{n}\right), x_{n}\right)_{p q}$. Then invoking hypothesis $\mathrm{H}(f)_{1}(\mathrm{v})$, we obtain

$$
\left\|x_{n}^{\prime}\right\|_{p}^{p} \leq k_{10}\left(\left\|\eta_{1, M}\right\|_{q}+\left\|\eta_{2, M}\right\|_{\infty}\left\|x_{n}^{\prime}\right\|_{p}^{p-1}\right)
$$

showing that $\left\{x_{n}^{\prime}\right\} \subseteq L^{p}\left(T, \mathbb{R}^{N}\right)$ is bounded, and so $\left\{x_{n}\right\} \subseteq W^{1, p}\left(T, \mathbb{R}^{N}\right)$ is bounded; hence we may assume $x_{n} \stackrel{w}{\rightarrow} x$ in $W^{1, p}\left(T, \mathbb{R}^{N}\right)$ and $x_{n} \rightarrow x$ in $L^{p}\left(T, \mathbb{R}^{N}\right)$. Also note that $\left\|A_{\lambda_{n}}\left(x_{n}(t)\right)\right\| \leq\left\|A^{0}\left(x_{n}(t)\right)\right\|$ (since $\operatorname{dom} A=\mathbb{R}^{N}$, see Hu-Papageorgiou [10], Proposition III.2.29, p. 325). Moreover, because $\operatorname{dom} A=\mathbb{R}^{N}, A^{0}$ is bounded on compact sets. Note that since $\left\{x_{n}\right\}_{n>1}$ is bounded in $W^{1, p}\left(T, \mathbb{R}^{N}\right)$, it is relatively compact in $C\left(T, \mathbb{R}^{N}\right)$. Hence, $\sup \left[\left\|A^{0}\left(x_{n}(t)\right)\right\|: t \in T, n \geq 1\right] \leq k_{11}$ for some $k_{11}>0$. Thus we may assume that $A_{\lambda_{n}}\left(x_{n}(\cdot)\right) \stackrel{w}{\rightarrow} u$ in $L^{q}\left(T, \mathbb{R}^{N}\right)$ as $n \rightarrow \infty$. Now arguing as in the proof of Proposition 4 (Claim 1), we see that $x_{n}^{\prime} \rightarrow x^{\prime}$ in $L^{p}\left(T, \mathbb{R}^{N}\right.$ ) and so $x_{n} \rightarrow x$ in $W^{1, p}\left(T, \mathbb{R}^{N}\right)$. Moreover, $\left\{\left\|x_{n}^{\prime}(\cdot)\right\|^{p-2} x_{n}^{\prime}(\cdot)\right\}_{n \geq 1} \subseteq W^{1, q}\left(T, \mathbb{R}^{N}\right)$ is bounded (since $\left(\left\|x_{n}^{\prime}(t)\right\|^{p-2} x_{n}^{\prime}(t)\right)^{\prime}=A_{\lambda_{n}}\left(x_{n}(t)\right)+f\left(t, x_{n}(t), x_{n}^{\prime}(t)\right)$ a.e. on $T)$. Thus we may assume that $\left\|x_{n}^{\prime}(\cdot)\right\|^{p-2} x_{n}^{\prime}(\cdot) \stackrel{w}{\rightarrow} v$ in $W^{1, q}\left(T, \mathbb{R}^{N}\right)$ and $\left\|x_{n}^{\prime}(\cdot)\right\|^{p-2} x_{n}^{\prime}(\cdot) \rightarrow v$ in $C\left(T, \mathbb{R}^{N}\right)$. Then $\varphi^{-1}\left(\left\|x_{n}^{\prime}(\cdot)\right\|^{p-2} x_{n}^{\prime}(\cdot)\right)=x_{n}^{\prime} \rightarrow$ $\varphi^{-1}(v)$ in $L^{p}\left(T, \mathbb{R}^{N}\right)$ and so $\varphi^{-1}(v)=x^{\prime}$ and $v=\left\|x^{\prime}(\cdot)\right\|^{p-2} x^{\prime}(\cdot)$. Therefore

$$
\left(\left\|x^{\prime}(t)\right\|^{p-2} x^{\prime}(t)\right)^{\prime}=u(t)+f\left(t, x(t), x^{\prime}(t)\right) \quad \text { a.e. on } T
$$


and $\left(\varphi\left(x^{\prime}(0)\right),-\varphi\left(x^{\prime}(b)\right) \in \xi(x(0), x(b))\right.$ (since $\mathrm{Gr} \xi$ is closed because of the maximal monotonicity of $\xi)$.

Now let $\widehat{A}: \widehat{D} \subseteq L^{p}\left(T, \mathbb{R}^{N}\right) \rightarrow L^{q}\left(T, \mathbb{R}^{N}\right)$ be defined by

$$
\widehat{A}(x)=\left\{g \in L^{q}\left(T, \mathbb{R}^{N}\right): g(t) \in A(x(t)) \text { a.e. on } T\right\}
$$

for all $x$ in

$$
\begin{aligned}
& \widehat{D}=\left\{x \in L^{p}\left(T, \mathbb{R}^{N}\right): \text { there is } g \in L^{q}\left(T, \mathbb{R}^{N}\right)\right. \text { satisfying } \\
& \qquad g(t) \in A(x(t)) \text { a.e. on } T\} .
\end{aligned}
$$

We claim that $\widehat{A}$ is maximal monotone. Since monotonicity is clear, we start to show that $R(\widehat{A}+J)=L^{q}\left(T, \mathbb{R}^{N}\right)$, where as before $J: L^{p}\left(T, \mathbb{R}^{N}\right) \rightarrow$ $L^{q}\left(T, \mathbb{R}^{N}\right)$ is defined by $J(x)(\cdot)=\left\|x^{\prime}(\cdot)\right\|^{p-2} x^{\prime}(\cdot)$. To this end let $h \in$ $L^{q}\left(T, \mathbb{R}^{N}\right)$ be given and let

$$
S(t)=\left\{(x, a) \in \mathbb{R}^{N} \times \mathbb{R}^{N}: a+\varphi(x)=h(t), a \in A(x),\|x\| \leq R(t)\right\}
$$

(we consider a finite-valued representative of $h$ ). The map $A+\varphi$ is maximal monotone (see Hu-Papageorgiou [10], Theorem III.3.3, p. 334) and coercive. Thus if we choose $R(t)=\|h(t)\|^{1 /(p-1)}+1$ large enough, then by Theorem III.6.28, p. 371, of Hu-Papageorgiou [10], we see that $S(t) \neq \emptyset$ for all $t \in T$. Moreover, $R \in L^{p}(T)_{+}$,

$\operatorname{Gr} S=\left\{(t, x, a) \in T \times \mathbb{R}^{N} \times \mathbb{R}^{N}: a+\varphi(x)=h(t), d(a, A(x))=0,\|x\| \leq R(t)\right\}$ (because $A(x)$ is closed (in fact compact), due to the maximality of $A$ ). So $\operatorname{Gr} S \in \mathcal{L} \times B\left(\mathbb{R}^{N}\right) \times B\left(\mathbb{R}^{N}\right)(\mathcal{L}=$ Lebesgue $\sigma$-field of $T)$. Invoking the Yankov-von Neumann-Aumann selection theorem (see $\mathrm{Hu}$-Papageorgiou [10], Theorem II.2.14, p. 158) we obtain measurable functions $x, a: T \rightarrow \mathbb{R}^{N}$ such that $(x(t), a(t)) \in S(t)$ a.e. on $T$, hence $a(t)+\varphi(x(t))=h(t)$ a.e. on $T$. Evidently $a \in L^{q}\left(T, \mathbb{R}^{N}\right)$. This proves the surjectivity of $\widehat{A}+J$.

Then we argue as in the proof of Proposition 3. Namely suppose that for $y \in L^{p}\left(T, \mathbb{R}^{N}\right)$ and $v \in L^{q}\left(T, \mathbb{R}^{N}\right)$ we have

$$
(a-v, x-y)_{p q} \geq 0 \quad \text { for all } x \in \widehat{D} \text { and all } a \in \widehat{A}(x) .
$$

Let $x=x_{1}$ where $x_{1} \in \widehat{D}$ is such that $v+J(y)=a_{1}+J\left(x_{1}\right), a_{1} \in \widehat{A}\left(x_{1}\right)$ (here we use the surjectivity of $\widehat{A}+J$ ). Then we can write

$$
\left(a_{1}-a_{1}-J\left(x_{1}\right)+J(y), x_{1}-y\right)_{p q} \geq 0,
$$

hence $\left(J(y)-J\left(x_{1}\right), x_{1}-y\right)_{p q} \geq 0$ and so $y=x_{1} \in \widehat{D}$ and $v=a_{1} \in \widehat{A}\left(x_{1}\right)$, which proves the maximality of $\widehat{A}$.

Recall that $A_{\lambda_{n}}\left(x_{n}(t)\right) \in A\left(J_{\lambda_{n}}\left(x_{n}(t)\right)\right)$ a.e. with $J_{\lambda_{n}}: \mathbb{R}^{N} \rightarrow \mathbb{R}^{N}$ being the resolvent operator corresponding to $A$. Note that

$$
\begin{aligned}
\left\|J_{\lambda_{n}}\left(x_{n}(t)\right)-x(t)\right\| & \leq\left\|J_{\lambda_{n}}\left(x_{n}(t)\right)-J_{\lambda_{n}}(x(t))\right\|+\left\|J_{\lambda_{n}}(x(t))-x(t)\right\| \\
& \leq\left\|x_{n}(t)-x(t)\right\|+\left\|J_{\lambda_{n}}(x(t))-x(t)\right\| \rightarrow 0
\end{aligned}
$$


as $n \rightarrow \infty$. Hence $J_{\lambda_{n}}\left(x_{n}(\cdot)\right) \rightarrow x(\cdot)$ in $L^{p}\left(T, \mathbb{R}^{N}\right)$ (dominated convergence theorem). Since $\left(J_{\lambda_{n}}\left(x_{n}(\cdot)\right), A_{\lambda_{n}}\left(x_{n}(\cdot)\right)\right) \in \mathrm{Gr} \widehat{A}$ and the latter is demiclosed (since $\widehat{A}$ is maximal monotone), we have $(x, u) \in \operatorname{Gr} \widehat{A}$, hence $u(t) \in A(x(t))$ a.e. on $T, u \in L^{q}\left(T, \mathbb{R}^{N}\right)$. This proves that $x \in C^{1}\left(T, \mathbb{R}^{N}\right)$ is a solution of (1).

Remark. An interesting byproduct of the above proof is that $\widehat{A}: D \subseteq$ $L^{p}\left(T, \mathbb{R}^{N}\right) \rightarrow 2^{L^{q}\left(T, \mathbb{R}^{N}\right)}$ is maximal monotone when $\operatorname{dom} A=\mathbb{R}^{N}$, a fact well known for $p=q=2$, but for which we have been unable to find a proof in the literature when $p \neq 2$.

Now we state and prove an existence theorem for problem (1) for the case when $\operatorname{dom} A$ is not all of $\mathbb{R}^{N}$.

TheOREM 6. If hypotheses $\mathrm{H}(A)_{2}, \mathrm{H}(f)_{2}, \mathrm{H}(\xi)$ and $\mathrm{H}_{0}$ hold, then problem (1) has a solution $x \in C^{1}\left(T, \mathbb{R}^{N}\right)$.

Proof. As in the proof of Theorem 5 , let $\lambda_{n} \rightarrow 0, \lambda_{n}>0$ and let $x_{n} \in C^{1}\left(T, \mathbb{R}^{N}\right)$ be the solutions of the auxiliary problem (7). Again $\left\{x_{n}\right\} \subseteq$ $W^{1, p}\left(T, \mathbb{R}^{N}\right)$ is bounded and so we may assume that $x_{n} \stackrel{w}{\rightarrow} x$ in $W^{1, p}\left(T, \mathbb{R}^{N}\right)$. Also for all $n \geq 1$ we have $V\left(x_{n}\right)+\widehat{A}_{\lambda_{n}}\left(x_{n}\right)=-N\left(x_{n}\right)$ and hence $\left(V\left(x_{n}\right)\right.$, $\left.\widehat{A}_{\lambda_{n}}\left(x_{n}\right)\right)_{p q}+\left\|\widehat{A}_{\lambda_{n}}\left(x_{n}\right)\right\|_{2}^{2}=-\left(N\left(x_{n}\right), \widehat{A}_{\lambda_{n}}\left(x_{n}\right)\right)_{p q}$.

Note that $\widehat{A}_{\lambda_{n}}\left(x_{n}\right) \in C\left(T, \mathbb{R}^{N}\right)$ for all $n \geq 1$. We have

$$
\begin{aligned}
\left(V\left(x_{n}\right), \widehat{A}_{\lambda_{n}}\left(x_{n}\right)\right)_{p q}= & -\int_{0}^{b}\left(\left(\left\|x_{n}^{\prime}(t)\right\|^{p-2} x_{n}^{\prime}(t)\right)^{\prime}, A_{\lambda_{n}}\left(x_{n}(t)\right)\right)_{\mathbb{R}^{N}} d t \\
= & -\left\|x_{n}^{\prime}(b)\right\|^{p-2}\left(x_{n}^{\prime}(b), A_{\lambda_{n}}\left(x_{n}(b)\right)\right)_{\mathbb{R}^{N}} \\
& +\left\|x_{n}^{\prime}(0)\right\|^{p-2}\left(x_{n}^{\prime}(0), A_{\lambda_{n}}\left(x_{n}(0)\right)\right)_{\mathbb{R}^{N}} \\
& +\int_{0}^{b}\left\|x_{n}^{\prime}(t)\right\|^{p-2}\left(x_{n}^{\prime}(t), \frac{d}{d t} A_{\lambda_{n}}\left(x_{n}(t)\right)\right)_{\mathbb{R}^{N}} d t
\end{aligned}
$$

(Green's identity). Since $A_{\lambda_{n}}(\cdot)$ is Lipschitz continuous, it is differentiable almost everywhere (Rademacher's theorem). Also from the monotonicity of $A_{\lambda_{n}}(\cdot)$ we have

$$
\left(y, \frac{A_{\lambda_{n}}(x+t y)-A_{\lambda_{n}}(x)}{t}\right)_{\mathbb{R}^{N}} \geq 0,
$$

i.e. $\left(y, A_{\lambda_{n}}^{\prime}(x) y\right)_{\mathbb{R}^{N}} \geq 0$ for every $x$ which is a point of differentiability of $A_{\lambda_{n}}(\cdot)$ and every $y \in \mathbb{R}^{N}$. Moreover, from Marcus-Mizel [13] we know that

$$
\frac{d}{d t} A_{\lambda_{n}}\left(x_{n}(t)\right)=A_{\lambda_{n}}^{\prime}\left(x_{n}(t)\right) x_{n}^{\prime}(t) \text {. }
$$


Then using hypothesis $\mathrm{H}_{0}$ and the property of $A_{\lambda_{n}}^{\prime}(\cdot)$ just proved, we obtain

$$
\left(V\left(x_{n}\right), \widehat{A}_{\lambda_{n}}\left(x_{n}\right)\right)_{p q} \geq 0 .
$$

Since $N\left(x_{n}\right) \in L^{2}\left(T, \mathbb{R}^{N}\right)$ (see hypothesis $\left.\mathrm{H}(f)_{2}\right)$, we can write

$$
\left\|\widehat{A}_{\lambda_{n}}\left(x_{n}\right)\right\|_{2}^{2} \leq\left\|N\left(x_{n}\right)\right\|_{2}\left\|\widehat{A}_{\lambda_{n}}\left(x_{n}\right)\right\|_{2}
$$

and thus $\left\{\widehat{A}_{\lambda_{n}}\left(x_{n}\right)\right\}_{n \geq 1} \subseteq L^{2}\left(T, \mathbb{R}^{N}\right) \subseteq L^{q}\left(T, \mathbb{R}^{N}\right)$ (since $q \leq 2 \leq p$ ) is bounded.

Thus we may assume that $\widehat{A}_{\lambda_{n}}\left(x_{n}\right) \stackrel{w}{\rightarrow} u$ in $L^{q}\left(T, \mathbb{R}^{N}\right)$. Also as in the proof of Theorem 5, we obtain $x_{n} \rightarrow x$ in $W^{1, p}\left(T, \mathbb{R}^{N}\right)$. Moreover, by the same argument as in that proof, $\left\|x_{n}^{\prime}(\cdot)\right\|^{p-2} x_{n}^{\prime}(\cdot) \stackrel{w}{\rightarrow}\left\|x^{\prime}(\cdot)\right\|^{p-2} x^{\prime}(\cdot)$ in $W^{1, q}\left(T, \mathbb{R}^{N}\right)$ and in the limit we have

$$
\left\{\begin{array}{l}
\left(\left\|x^{\prime}(t)\right\|^{p-2} x^{\prime}(t)\right)^{\prime}=u(t)+f\left(t, x(t), x^{\prime}(t)\right) \quad \text { a.e. on } T, \\
\left(\varphi\left(x^{\prime}(0)\right),-\varphi\left(x^{\prime}(b)\right)\right) \in \xi(x(0), x(b)) .
\end{array}\right.
$$

To finish the proof we need to show that $u(t) \in A(x(t))$ a.e. on $T$. To this end let $J_{\lambda}: \mathbb{R}^{N} \rightarrow \mathbb{R}^{N}$ be the resolvent of $A$ and $\widehat{J}_{\lambda}: L^{p}\left(T, \mathbb{R}^{N}\right) \rightarrow$ $L^{p}\left(T, \mathbb{R}^{N}\right)$ the corresponding Nemytskiu operator, i.e. $J_{\lambda}(x)(\cdot)=J_{\lambda}(x(\cdot))$ (recall that $J_{\lambda}(\cdot)$ is nonexpansive). By Marcus-Mizel [13] we know that for every $n \geq 1, J_{\lambda_{n}}\left(x_{n}(\cdot)\right) \in W^{1, p}\left(T, \mathbb{R}^{N}\right)$ and

$$
\frac{d}{d t} J_{\lambda_{n}}\left(x_{n}(t)\right)=J_{\lambda_{n}}^{\prime}\left(x_{n}(t)\right) x_{n}^{\prime}(t), \quad\left\|J_{\lambda_{n}}^{\prime}\left(x_{n}(t)\right)\right\| \leq 1 .
$$

Thus $\left\|J_{\lambda_{n}}^{\prime}\left(x_{n}(t)\right) x_{n}^{\prime}(t)\right\| \leq\left\|x_{n}^{\prime}(t)\right\|$ a.e. on $T$, from which it follows that $\left\{J_{\lambda_{n}}\left(x_{n}(\cdot)\right)=\widehat{J}_{\lambda_{n}}\left(x_{n}\right)\right\}_{n \geq 1} \subseteq W^{1, p}\left(T, \mathbb{R}^{N}\right.$ ) is bounded (note $J_{\lambda_{n}}(0)=0$ ). Thus we may assume that $\widehat{J}_{\lambda_{n}}\left(x_{n}\right) \stackrel{w}{\rightarrow} z$ in $W^{1, p}\left(T, \mathbb{R}^{N}\right)$ and $\widehat{J}_{\lambda_{n}}\left(x_{n}\right) \rightarrow z$ in $C\left(T, \mathbb{R}^{N}\right)$. We know that

$$
J_{\lambda_{n}}\left(x_{n}(t)\right)+\lambda_{n} A_{\lambda_{n}}\left(x_{n}(t)\right)=x_{n}(t) \quad\left(\text { since } A_{\lambda_{n}}=\lambda_{n}^{-1}\left(I-J_{\lambda_{n}}\right)\right),
$$

and so $\widehat{J}_{\lambda_{n}}\left(x_{n}\right)+\lambda_{n} \widehat{A}_{\lambda_{n}}\left(x_{n}\right)=x_{n}$. Passing to the limit in the above equality and since $\lambda_{n} \rightarrow 0$ and $\left\{\widehat{A}_{\lambda_{n}}\left(x_{n}\right)\right\}_{n \geq 1} \subseteq L^{q}\left(T, \mathbb{R}^{N}\right)$ is bounded, we obtain $z=x$. So $\widehat{J}_{\lambda_{n}}\left(x_{n}\right) \rightarrow x$ in $C\left(T, \mathbb{R}^{N}\right)$.

Now let

$\Gamma=\left\{t \in T\right.$ : there exist $y \in \mathbb{R}^{N}$ and $v \in A(y)$ such that

$$
\left.(u(t)-v, x(t)-y)_{\mathbb{R}^{N}}<0\right\} .
$$

If we show that $\Gamma \subseteq T$ is a Lebesgue-null set, then from the maximal monotonicity of $A(\cdot)$ we will have $u(t) \in A(x(t))$ a.e. on $T$. In what follows, we denote by $|\cdot|$ the Lebesgue measure on $T$. Let $E: T \rightarrow 2^{\mathbb{R}^{N} \times \mathbb{R}^{N}}$ be defined by $E(t)=\left\{(y, v) \in \mathbb{R}^{N} \times \mathbb{R}^{N}: v \in A(y),(u(t)-v, x(t)-y)_{\mathbb{R}^{N}}<0\right\}$. Evidently $\Gamma=\operatorname{dom} E=\{t \in T: E(t) \neq \emptyset\}$. Note that $\operatorname{Gr} E=\{(t, y, v) \in$ $\left.T \times \mathbb{R}^{N} \times \mathbb{R}^{N}: d(v, A(y))=0, \vartheta(t, y, v)<0\right\}$, where $\vartheta(t, y, v)=(u(t)-$ 
$v, x(t)-y)_{\mathbb{R}^{N}}$ (recall that $A(\cdot)$ is closed valued). Since $\vartheta$ is measurable in $t$ and continuous in $(y, v)$, it is jointly measurable (see $\mathrm{Hu}-\mathrm{Papageorgiou} \mathrm{[10],}$ Proposition II.1.6, p. 142) and so $\operatorname{Gr} E \in \mathcal{L} \times B\left(\mathbb{R}^{N}\right) \times B\left(\mathbb{R}^{N}\right)$. Then from the Yankov-von Neumann-Aumann projection theorem (see $\mathrm{Hu}-\mathrm{Papageorgiou}$ [10], Theorem II.1.33, p. 149), we have $\operatorname{proj}_{T} \operatorname{Gr} E=\operatorname{dom} E \in \mathcal{L}$.

Suppose $|\Gamma|>0$. Apply the Yankov-von Neumann-Aumann selection theorem (see Hu-Papageorgiou [10], Theorem II.2.14, p. 158) to obtain measurable maps $y: \Gamma \rightarrow \mathbb{R}^{N}, v: \Gamma \rightarrow \mathbb{R}^{N}$ such that $(y(t), v(t)) \in E(t)$ for all $t \in \Gamma$. By Lusin's theorem we can find closed $\Gamma_{1} \subseteq \Gamma$ with $\left|\Gamma_{1}\right|>0$ such that $\left.y\right|_{\Gamma_{1}},\left.v\right|_{\Gamma_{1}}$ are continuous, hence bounded. Recall that $A_{\lambda_{n}}\left(x_{n}(t)\right) \in$ $A\left(J_{\lambda_{n}}\left(x_{n}(t)\right)\right)$ and so

$$
\left(A_{\lambda_{n}}\left(x_{n}(t)\right)-v(t), J_{\lambda_{n}}\left(x_{n}(t)\right)-y(t)\right)_{\mathbb{R}^{N}} \geq 0 \quad \text { on } \Gamma_{1},
$$

hence

$$
\int_{\Gamma_{1}}\left(A_{\lambda_{n}}\left(x_{n}(t)\right)-v(t), J_{\lambda_{n}}\left(x_{n}(t)\right)-y(t)\right)_{\mathbb{R}^{N}} d t \geq 0
$$

and therefore

$$
\int_{\Gamma_{1}}(u(t)-v(t), x(t)-y(t))_{\mathbb{R}^{N}} d t \geq 0 .
$$

But since $\left|\Gamma_{1}\right|>0$ and $(y(t), v(t)) \in E(t)$ for all $t \in \Gamma$, we have

$$
\int_{\Gamma_{1}}(u(t)-v(t), x(t)-y(t))_{\mathbb{R}^{N}} d t<0,
$$

a contradiction. Thus $|\Gamma|=0$ and so $u(t) \in A(x(t))$ a.e. on $T$. This then proves that $x \in C^{1}\left(T, \mathbb{R}^{N}\right)$ solves the problem.

We conclude this section with some important special cases of problem (1).

(a) Let $K_{1}, K_{2} \subseteq \mathbb{R}^{N}$ be nonempty, closed and convex with $0 \in K_{1} \cap K_{2}$. Let $\delta_{K_{1} \times K_{2}}$ be the indicator function of the convex set $K_{1} \times K_{2}$, i.e.

$$
\delta_{K_{1} \times K_{2}}(x, y)= \begin{cases}0 & \text { if }(x, y) \in K_{1} \times K_{2}, \\ +\infty & \text { otherwise }\end{cases}
$$

and $\xi=\partial \delta_{K_{1} \times K_{2}}=N_{K_{1} \times K_{2}}=N_{K_{1}} \times N_{K_{2}}$ (for $C \subseteq \mathbb{R}^{N}$ nonempty, $N_{C}$ denotes the normal cone to $C$, see Hu-Papageorgiou [10], Definition VI.5.1, p. 624 and Proposition VI.5.39, pp. 636-637). Then problem (1) becomes

$$
\left\{\begin{array}{l}
\left(\left\|x^{\prime}(t)\right\|^{p-2} x^{\prime}(t)\right)^{\prime} \in A(x(t))+f\left(t, x(t), x^{\prime}(t)\right), \\
x(0) \in K_{1}, \quad x(b) \in K_{2}, \\
\left(x^{\prime}(0), x(0)\right)_{\mathbb{R}^{N}}=\sigma\left(x^{\prime}(0), K_{1}\right), \\
\left(-x^{\prime}(b), x(b)\right)_{\mathbb{R}^{N}}=\sigma\left(-x^{\prime}(b), K_{2}\right) .
\end{array}\right.
$$

Note that $\xi=\partial \delta_{K_{1} \times K_{2}}$ is maximal monotone, $(0,0) \in \xi(0,0)$ and from the definition of the normal cone we see that $\left(a^{\prime}, d^{\prime}\right) \in \xi(a, d)=N_{K_{1}}(a) \times N_{K_{2}}(d)$ 
implies $\left(a^{\prime}, a\right)_{\mathbb{R}^{N}} \geq 0,\left(d^{\prime}, d\right)_{\mathbb{R}^{N}} \geq 0$ since $0 \in K_{1} \cap K_{2}$. So hypothesis $\mathrm{H}(\xi)$ (with (i) in effect) holds. Thus we can state the following corollary.

Corollary 7. If $\mathrm{H}(A)_{1}, \mathrm{H}(f)_{1}$ (or $\mathrm{H}(A)_{2}, \mathrm{H}(f)_{2}$ and $\left.\mathrm{H}_{0}\right)$ hold, then problem (12) has a solution $x \in C^{1}\left(T, \mathbb{R}^{N}\right)$.

In particular suppose that $K_{1}, K_{2} \subseteq \mathbb{R}_{+}^{N}$ and let $A=\partial \psi$ where $\psi=\delta_{\mathbb{R}_{+}^{N}}$. Then

$$
\begin{aligned}
A(x) & =N_{\mathbb{R}_{+}^{N}}(x) \\
& = \begin{cases}\{0\} & \text { if } x_{i}>0 \text { for all }\{i \in 1, \ldots, N\}, \\
-\mathbb{R}_{+}^{N} \cap\{x\}^{\perp} & \text { if there is } i \in\{1, \ldots, N\} \text { such that } x_{i}=0,\end{cases}
\end{aligned}
$$

for $x \in \mathbb{R}_{+}^{N}$. Also it is easy to see that $A_{\lambda}(x)=\lambda^{-1}\left(x-p\left(x ; \mathbb{R}_{+}^{N}\right)\right), \lambda>0$, where $p\left(\cdot ; \mathbb{R}_{+}^{N}\right)$ denotes the metric projection on $\mathbb{R}_{+}^{N}$. Then for $x \in K_{1}$ or $x \in K_{2}, p\left(x ; \mathbb{R}_{+}^{N}\right)=x$ since $A_{\lambda}(x)=0$ and so hypothesis $\mathrm{H}_{0}$ holds. Thus by Corollary 7 we are guaranteed a solution $x \in C^{1}\left(T, \mathbb{R}^{N}\right)$ for the following problem, provided $f$ satisfies $\mathrm{H}(f)_{2}$ :

$$
\left\{\begin{array}{c}
\left(\left\|x^{\prime}(t)\right\|^{p-2} x^{\prime}(t)\right)^{\prime}=f\left(t, x(t), x^{\prime}(t)\right) \quad \text { a.e. } \\
\quad \text { on }\left\{t \in T: x_{i}(t)>0 \text { for all } i \in\{1, \ldots, N\}\right\}, \\
\left(\left\|x^{\prime}(t)\right\|^{p-2} x^{\prime}(t)\right)^{\prime} \leq f\left(t, x(t), x^{\prime}(t)\right)-u(t) \text { a.e. } \\
\quad \text { on }\left\{t \in T: x_{i}(t)=0 \text { for some } i \in\{1, \ldots, N\}\right\} \\
u(t) \geq 0, \quad(x(t), u(t))_{\mathbb{R}^{N}}=0, \\
x(t) \geq 0 \text { for all } t \in T, x(0) \in K_{1}, \quad x(b) \in K_{2}, \\
\left(x^{\prime}(0), x(0)\right)_{\mathbb{R}^{N}}=\sigma\left(x^{\prime}(0), K_{1}\right), \quad\left(-x^{\prime}(b), x(b)\right)_{\mathbb{R}^{N}}=\sigma\left(-x^{\prime}(b), K_{2}\right) .
\end{array}\right.
$$

Above, the ordering on $\mathbb{R}^{N}$ is the usual componentwise partial ordering. Also observe that the solution $x$ remains in $\operatorname{dom} A=\mathbb{R}_{+}^{N} \varsubsetneqq \mathbb{R}^{N}$ (in other words the special choice of the maximal monotone part $A$ translates into a viability result for the convex set $\mathbb{R}_{+}^{N}$ ).

(b) In case (a) above, let $K_{1}=K_{2}=\{0\}$. Then problem (1) becomes the usual Dirichlet problem:

$$
\left\{\begin{array}{l}
\left(\left\|x^{\prime}(t)\right\|^{p-2} x^{\prime}(t)\right)^{\prime} \in A(x(t))+f\left(t, x(t), x^{\prime}(t)\right) \quad \text { a.e. on } T \\
x(0)=x(b)=0
\end{array}\right.
$$

Since $A_{\lambda}(0)=0$, we see that hypothesis $\mathrm{H}_{0}$ holds. Thus we can state the following existence result for problem (13).

Corollary 8. If hypotheses $\mathrm{H}(A)_{1}, \mathrm{H}(f)_{1}$ (or $\left.\mathrm{H}(A)_{2}, \mathrm{H}(f)_{2}\right)$ hold, then problem (13) has a solution $x \in C^{1}\left(T, \mathbb{R}^{N}\right)$.

(c) In example (a), let $K_{1}=K_{2}=\mathbb{R}^{N}$. Then problem (1) becomes the usual Neumann problem:

$$
\left\{\begin{array}{l}
\left(\left\|x^{\prime}(t)\right\|^{p-2} x^{\prime}(t)\right)^{\prime} \in A(x(t))+f\left(t, x(t), x^{\prime}(t)\right) \quad \text { a.e. on } T \\
x^{\prime}(0)=x^{\prime}(b)=0 .
\end{array}\right.
$$


Since $\partial \delta_{K_{1} \times K_{2}}=N_{K_{1}} \times N_{K_{2}}=\{(0,0)\}$, we see that hypothesis $\mathrm{H}_{0}$ is trivially fulfilled. So the following existence result is true for problem (14).

Corollary 9. If hypotheses $\mathrm{H}(A)_{1}, \mathrm{H}(f)_{1}$ (or $\left.\mathrm{H}(A)_{2}, \mathrm{H}(f)_{2}\right)$ hold, then problem (14) has a solution $x \in C^{1}\left(T, \mathbb{R}^{N}\right)$.

(d) Let $K=\left\{(x, y) \in \mathbb{R}^{N} \times \mathbb{R}^{N}: x=y\right\}$ and let $\xi=\partial \delta_{K}$ be the indicator function for $K$. Note that $K \subseteq \mathbb{R}^{N} \times \mathbb{R}^{N}$ is a subspace and so $\partial \delta_{K}=K^{\perp}=\left\{(v, w) \in \mathbb{R}^{N} \times \mathbb{R}^{N}: v=-w\right\}$. Problem (1) becomes the usual periodic problem:

$$
\left\{\begin{array}{l}
\left(\left\|x^{\prime}(t)\right\|^{p-2} x^{\prime}(t)\right)^{\prime} \in A(x(t))+f\left(t, x(t), x^{\prime}(t)\right) \quad \text { a.e. on } T, \\
x(0)=x(b), \quad x^{\prime}(0)=x^{\prime}(b) .
\end{array}\right.
$$

In this case hypothesis $\mathrm{H}(\xi)$ (ii) is satisfied. Also if $\left(a^{\prime}, d^{\prime}\right) \in \xi(a, d)$, we have $a=d$ and $a^{\prime}=-d^{\prime}$ and so $\left(A_{\lambda}(a), a^{\prime}\right)_{\mathbb{R}^{N}}+\left(A_{\lambda}(d), d^{\prime}\right)_{\mathbb{R}^{N}}=0$, hence hypothesis $\mathrm{H}_{0}$ holds. Thus we can state the following existence result for problem (15).

Corollary 10. If hypotheses $\mathrm{H}(A)_{1}, \mathrm{H}(f)_{1}$ (or $\left.\mathrm{H}(A)_{2}, \mathrm{H}(f)_{2}\right)$ hold, then problem (15) has a solution $x \in C^{1}\left(T, \mathbb{R}^{N}\right)$.

(e) Let $\xi: \mathbb{R}^{N} \times \mathbb{R}^{N} \rightarrow \mathbb{R}^{N} \times \mathbb{R}^{N}$ be defined by

$$
\xi(x, y)=\left(\frac{1}{\vartheta^{p-1}} \varphi(x), \frac{1}{\eta^{p-1}} \varphi(y)\right),
$$

for some $\vartheta, \eta>0$. Then $\xi$ is monotone, continuous (thus maximal monotone) and $\xi(0,0)=(0,0)$. Problem (1) becomes the following Sturm-Liouville-type problem:

$$
\left\{\begin{array}{l}
\left(\left\|x^{\prime}(t)\right\|^{p-2} x^{\prime}(t)\right)^{\prime} \in A(x(t))+f\left(t, x(t), x^{\prime}(t)\right) \quad \text { a.e. on } T \\
x(0)-\vartheta x^{\prime}(0)=0, \quad x(b)+\eta x^{\prime}(b)=0 .
\end{array}\right.
$$

Note that if $\left(a^{\prime}, d^{\prime}\right)=\xi(a, d)$, then $\left(a^{\prime}, a\right)_{\mathbb{R}^{N}}=\vartheta^{1-p}\|a\|^{p} \geq 0$ and $\left(d^{\prime}, d\right)_{\mathbb{R}^{N}}=\eta^{1-p}\|d\|^{p} \geq 0$, which means that hypothesis $\mathrm{H}(\xi)(\mathrm{i})$ is satisfied. Also $\left(A_{\lambda}(a), a^{\prime}\right)_{\mathbb{R}^{N}}=\left(A_{\lambda}(a), \vartheta^{1-p}\|a\|^{p-2} a\right)_{\mathbb{R}^{N}}=\vartheta^{1-p}\|a\|^{p-2}\left(A_{\lambda}(a), a\right)_{\mathbb{R}^{N}}$ $\geq 0$ because $A_{\lambda}(\cdot)$ is monotone and $A_{\lambda}(0)=0$. Similarly $\left(A_{\lambda}(d), d^{\prime}\right)_{\mathbb{R}^{N}} \geq 0$. Therefore hypothesis $\mathrm{H}_{0}$ holds and we can state the following existence result.

Corollary 11. If hypotheses $\mathrm{H}(A)_{1}, \mathrm{H}(f)_{1}$ (or $\left.\mathrm{H}(A)_{2}, \mathrm{H}(f)_{2}\right)$ hold, then problem (16) has a solution.

(f) Let $\xi: \mathbb{R}^{N} \times \mathbb{R}^{N} \rightarrow \mathbb{R}^{N} \times \mathbb{R}^{N}$ be defined by $\xi(x, y)=\left(x-g_{1}(x), y-\right.$ $\left.g_{2}(y)\right)$, with $g_{1}, g_{2}: \mathbb{R}^{N} \rightarrow \mathbb{R}^{N}$ nonexpansive maps with $g_{1}(0)=g_{2}(0)=0$. Then $\xi$ is monotone continuous (thus maximal monotone) and $\xi(0,0)=$ $(0,0)$. Also assume that $\left(g_{i}(x), x\right)_{\mathbb{R}^{N}} \leq c\|x\|^{2}$ for all $x \in \mathbb{R}^{N}$ and $i=1,2$, 
with $0<c<1$. Suppose that for all $x \in \mathbb{R}^{N}$ and all $\lambda>0,\left(A_{\lambda}(x), g_{i}(x)\right)_{\mathbb{R}^{N}}$ $\leq\left(A_{\lambda}(x), x\right)_{\mathbb{R}^{N}}$ for $i=1,2$. Then hypothesis $\mathrm{H}_{0}$ holds. The problem now is the following:

$$
\left\{\begin{array}{l}
\left(\left\|x^{\prime}(t)\right\|^{p-2} x^{\prime}(t)\right)^{\prime} \in A(x(t))+f\left(t, x(t), x^{\prime}(t)\right) \quad \text { a.e. on } T, \\
\left\|x^{\prime}(0)\right\|^{p-2} x^{\prime}(0)=x(0)-g_{1}(x(0)), \\
-\left\|x^{\prime}(b)\right\|^{p-2} x^{\prime}(b)=x(b)-g_{2}(x(b)) .
\end{array}\right.
$$

We can state the following result concerning problem (17):

Corollary 12. If hypotheses $\mathrm{H}(A)_{1}, \mathrm{H}(f)_{1}$ (or $\left.\mathrm{H}(A)_{2}, \mathrm{H}(f)_{2}\right)$ hold and $\xi(x, y)$ is as above, then problem (17) has a solution $x \in C^{1}\left(T, \mathbb{R}^{N}\right)$.

\section{References}

[1] R. Adams, Sobolev Spaces, Academic Press, New York, 1975.

[2] L. Boccardo, P. Drábek, D. Giachetti and M. Kučera, Generalization of Fredholm alternative for nonlinear differential operators, Nonlinear Anal. 10 (1986), 1083-1103.

[3] H. Dang and S. F. Oppenheimer, Existence and uniqueness results for some nonlinear boundary value problems, J. Math. Anal. Appl. 198 (1996), 35-48.

[4] M. del Pino, M. Elgueta and R. Manásevich, A homotopic deformation along $p$ of a Leray-Schauder degree result and existence for $\left(\left|u^{\prime}\right|^{p-2} u^{\prime}\right)^{\prime}+f(t, u)=0, u(0)=$ $u(T)=0, p>1$, J. Differential Equations 80 (1989), 1-13.

[5] M. del Pino, R. Manásevich and A. Murúa, Existence and multiplicity of solutions with prescribed period for a second order quasilinear o.d.e., Nonlinear Anal. 18 (1992), 79-92.

[6] C. Fabry and D. F ayy ad, Periodic solutions of second order differential equations with a p-Laplacian and asymmetric nonlinearities, Rend. Istit. Mat. Univ. Trieste 24 (1992), 207-227.

[7] Z. Guo, Boundary value problems of a class of quasilinear ordinary differential equations, Differential Integral Equations 6 (1993), 705-719.

[8] P. Hartman, Ordinary Differential Equations, Wiley, New York, 1964.

[9] E. Hewitt and K. Stromberg, Real and Abstract Analysis, Springer, New York, 1965.

[10] S. Hu and N. S. Papageorgiou, Handbook of Multivalued Analysis. Volume I: Theory, Kluwer, Dordrecht, 1997.

[11] D. Kandilakis and N. S. Papageorgiou, Neumann problem for a class of quasilinear ordinary differential equations, Atti Sem. Mat. Fis. Univ. Modena, to appear.

[12] R. Manásevich and J. Mawhin, Periodic solutions for nonlinear systems with p-Laplacian-like operators, J. Differential Equations 145 (1998), 367-393.

[13] M. Marcus and V. Mizel, Absolute continuity on tracks and mappings of Sobolev spaces, Arch. Rational Mech. Anal. 45 (1972), 294-320.

[14] J. Mawhin and M. Willem, Critical Point Theory and Hamiltonian Systems, Springer, New York, 1989. 
[15] E. Zeidler, Nonlinear Functional Analysis and its Applications I, Springer, New York, 1985.

[16] - Nonlinear Functional Analysis and its Applications II, Springer, New York, 1990.

Center for Mathematical Sciences

Munich University of Technology (TUM)

Arcisstr. 21

D-80333 München, Germany

E-mail: bader@appl-math.tu-muenchen.de
Department of Mathematics National Technical University Zografou Campus Athens 15780, Greece E-mail: npapg@math.ntua.gr

Reçu par la Rédaction le 20.7.1999 